\begin{tabular}{|c|l|}
\hline Title & Chaotic Motion of the N-V ortex Problem on a Sphere: I. Saddle-Centers in Two-Degree of-Freedom Hamiltonians \\
\hline Author(s) & Sakajo, Takashi; Y agasaki, Kazuy uki \\
\hline Citation & $\begin{array}{l}\text { Journal of Nonlinear Science, 18, 485-525 } \\
\text { https://doi.org/10.1007/300332-008-9019-9 }\end{array}$ \\
\hline Issue Date & 2008-03-04 \\
\hline Doc URL & http://hdl.handle.net/2115/34776 \\
\hline Rights & The original publication is available at www.springerlink.com \\
\hline Type & article (author version) \\
\hline File Information & hsc8.pdf \\
\hline
\end{tabular}

Instructions for use 


\title{
Chaotic motion of the $N$-vortex problem on a sphere : I. Saddle-centers in two-degree-of-freedom Hamiltonians
}

\author{
Takashi SAKAJO \\ Department of Mathematics, Hokkaido University, \\ Sapporo Hokkaido 060-8610, JAPAN. \\ E-mail : sakajo@math.sci.hokudai.ac.jp. \\ Kazuyuki YAGASAKI \\ Department of Mechanical and Systems Engineering, Gifu University, \\ Gifu 501-1193, JAPAN \\ E-mail : yagasaki@gifu-u.ac.jp
}

January 31, 2008

\begin{abstract}
We study the motion of $N$ point vortices with $N \in \mathbb{N}$ on a sphere in the presence of fixed pole vortices, which are governed by a Hamiltonian dynamical system with $N$ degrees of freedom. Special attention is paid to the evolution of their polygonal ring configuration called the $N$-ring, in which they are equally spaced along a line of latitude of the sphere. When the number of the point vortices is $N=5 n$ or $6 n$ with $n \in \mathbb{N}$, the system is reduced to a two-degree-of-freedom Hamiltonian with some saddle-center equilibria, one of which corresponds to the unstable $N$-ring. Utilizing a Melnikov-type method applicable to two-degree-of-freedom Hamiltonian systems with saddle-center equilibria and a numerical method to compute stable and unstable manifolds, we show numerically that there exist transverse homoclinic orbits to unstable periodic orbits in the neighborhood of the saddle-centers and hence chaotic motions occur. Especially, the evolution of the unstable $N$-ring is shown to be chaotic.
\end{abstract}

PACS: 47.32.C-,47.52.+j,47.20.Ky

Keywords: Hamiltonian system, point vortex, flow on sphere, chaos, Melnikov method

\section{Introduction}

We consider an incompressible and inviscid fluid flow on a sphere, which is often used as a model of planetary flows. Since the vorticity, which is defined as the curl of the velocity field, is conserved along the path of a fluid particle, a point on the sphere with non-zero vorticity evolves as if it were a material point, which is called a point vortex. Thus for a given initial non-zero vorticity 
region, approximating it with a set of the $N$ point vortices, we can reduce the Euler equations to $2 N$-dimensional ordinary differential equations, which are referred to as the $N$-vortex problem. The $N$-vortex problem on the sphere has been studied by many researchers when $N$ is small. For instance, the 3vortex problem is integrable and its motion has been described in detail [18, 35]. A collapsing of three point vortices was found in [19]. An integrable 4vortex problem has been solved recently [41]. Relative fixed configurations with special symmetries, where the $N$ point vortices never change their shape, were investigated in a systematic way [26]. Many references of this subject are found in the books of Lim et. al [27] and Newton [30].

Let $\left(\Theta_{m}, \Psi_{m}\right)$ denote the position of the $m$ th point vortex in the spherical coordinates. We assume that all the point vortices have an identical strength, which is fixed to the unity without loss of generality. Then equations of motion for the $N$ point vortices are given by

$$
\begin{aligned}
\dot{\Theta}_{m}= & -\frac{1}{4 \pi} \sum_{j \neq m}^{N} \frac{\sin \Theta_{j} \sin \left(\Psi_{m}-\Psi_{j}\right)}{1-\cos \Theta_{m} \cos \Theta_{j}-\sin \Theta_{m} \sin \Theta_{j} \cos \left(\Psi_{m}-\Psi_{j}\right)} \\
\dot{\Psi}_{m}= & -\frac{1}{4 \pi \sin \Theta_{m}} \sum_{j \neq m}^{N} \frac{\cos \Theta_{m} \sin \Theta_{j} \cos \left(\Psi_{m}-\Psi_{j}\right)-\sin \Theta_{m} \cos \Theta_{j}}{1-\cos \Theta_{m} \cos \Theta_{j}-\sin \Theta_{m} \sin \Theta_{j} \cos \left(\Psi_{m}-\Psi_{j}\right)} \\
& +\frac{\Gamma_{1}}{4 \pi} \frac{1}{1-\cos \Theta_{m}}-\frac{\Gamma_{2}}{4 \pi} \frac{1}{1+\cos \Theta_{m}}, \quad m=1,2, \ldots, N
\end{aligned}
$$

The last two forcing terms in (1.2) represent the flow fields induced by special point vortices that are fixed at the north and the south poles, whose strengths are denoted by $\Gamma_{1}$ and $\Gamma_{2}$ respectively. They are formally introduced in order to incorporate a local effect of rotation of the sphere. While the point-vortex model cannot rigorously represent the motion of coherent vortex structures on the rotating sphere since the vorticity is no longer a conserved quantity, it is often valid to regard the effect of rotation as an interaction between the point vortices and uniform vorticity strips corresponding to the solid-body rotation [31,34]. However, the model is difficult to treat mathematically even with assistance of numerical methods. Our pole-vortices model is a simpler one in which the motion of point vortices is studied under the influence of an outer flow that resembles the local effect of rotation.

Equations (1.1) and (1.2) define a dynamical system in the $2 N$-dimensional phase space $\mathbb{P}_{N}$,

$$
\boldsymbol{X}=\left(\Theta_{1}, \Theta_{2}, \ldots, \Theta_{N}, \Psi_{1}, \Psi_{2}, \ldots, \Psi_{N}\right) \in \mathbb{P}_{N} \equiv[0, \pi]^{N} \times(\mathbb{R} / 2 \pi \mathbb{Z})^{N}
$$

Solutions of (1.1) and (1.2) exist globally in time since self-similar collapse of identical point vortices never occurs [19]. As we will see in $\S 3$, the dynamical system can be rewritten as a Hamiltonian system whose Hamiltonian is 
represented by

$$
\begin{aligned}
H= & -\frac{1}{4 \pi} \sum_{m=1}^{N} \sum_{m<j}^{N} \log \left(1-\cos \Theta_{m} \cos \Theta_{j}-\sin \Theta_{m} \sin \Theta_{j} \cos \left(\Psi_{m}-\Psi_{j}\right)\right) \\
& -\frac{\Gamma_{1}}{4 \pi} \sum_{m=1}^{N} \log \left(1-\cos \Theta_{m}\right)-\frac{\Gamma_{2}}{4 \pi} \sum_{m=1}^{N} \log \left(1+\cos \Theta_{m}\right)
\end{aligned}
$$

in the $X$-coordinates (see also Ref. [30]). In particular, the Hamiltonian (1.3) is constant along solutions of (1.1) and (1.2). We note that the quantity $I=$ $\sum_{m=1}^{N} \cos \Theta_{m}$ is also invariant due to the invariance of the Hamiltonian with respect to the rotation around the $z$-axis.

Here we pay special attention to a polygonal ring configuration of the $N$ point vortices aligned at a line of latitude of the sphere, called the $N$-ring, when $N$ is not small. Such a polygonal alignment of coherent vortex structures often appears in numerical simulations of planetary flows $[10,34,36]$, but their interaction is too complicated to track their evolutions for a longer time. While the $N$-ring is one of the simplest models to approximate the evolution of such coherent structures, it is also one of relative equilibria of (1.1) and (1.2) discussed in [26]. Linear and nonlinear stability of the $N$-ring has been extensively investigated $[3,5,20,37]$.

A goal of the present study is to describe how the $N$-ring evolves when it becomes unstable under the influence of the pole vortices. It is generally difficult to describe the evolution of the $N$ point vortices for large $N$, since the dimension of the phase space $\mathbb{P}_{N}$ becomes quite large. Although Lim showed that a general class of Hamiltonians with logarithmic singularities including (1.3) can be transformed to near-integrable Hamiltonians in small regions of the phase spaces near vortex clustering configurations in Ref. [23] or [24,25], we cannot appeal to such an approach since the vortex motions are rather far from the clustering configurations. One treatment for the difficulty is to reduce the high-dimensional system to a low-dimensional one, in which orbits are regarded as those embedded in the full system. That is to say, if a chaotic solution were found in the reduced system, it would really exist even in the original non-restricted system.

In the present paper, with the systematic reduction method given in [39], the $N$-vortex problem on the sphere can be reduced to two-degree-of-freedom Hamiltonian systems for $N=5 n$ and $6 n$ with $n \in \mathbb{N}$. For infinite lattices of point vortices on the plane, Lim [22] assumed a simple periodic structure to reduce the problem to a two-degree-of-freedom Hamiltonian system and proved the existence of quasi-periodic KAM tori in the reduced system. Our reduction approach does not assume such a simple structure in the motion of point vortices. In the reduced two-degree-of-freedom Hamiltonian systems, the unstable $N$-ring acquires a special property: The linear stability analysis [37] shows that the $N$-ring corresponds to a saddle-center equilibrium in some cases when it becomes unstable. That is to say, among the four eigenvalues of the linearized system for the $N$-ring, the two are real, and the other two are purely imaginary. According to the Lyapunov center theorem [28], there exist a one-parameter 
family of unstable periodic orbits, which may have transverse homoclinic orbits yielding chaotic dynamics $[13,45]$, around the saddle-center. We show that this type of chaos occurs in the reduced systems, by applying a Melnikov-type technique [46] for $N=6$ and by using a numerical technique of [50] for $N=5$ as well as $N=6$, where both analyses are carried out with assistance of a standard computer software called "AUTO" [7]. The numerical computations performed in the Melnikov analysis are considered very accurate, as shown in Appendix B for a generalized version of the Hénon-Heiles system [16]. We should also note that the invariant manifolds (spaces) on which the two-degree-of-freedom reduced systems exist are unstable as seen in $\S 2$, but if chaotic motions occur in the reduced systems, then similar motions can also occur in the full system by the Poincaré recurrence theorem [1]. See $\S 7$ for the details.

The Melnikov analysis is a familiar method to prove the existence of transverse homo- or heteroclinic orbits to periodic orbits in time-periodic perturbations of single-degree-of-freedom Hamiltonian systems $[13,45]$. It has also been extended in several directions (see, e.g., Refs. [15,43,44] and references therein) but almost all of such extensions are still limited to perturbations of integrable or partially integrable systems with homo- or heteroclinic orbits. For example, Lim [25] used such an extension of Melnikov's method [17] to analyze Arnold diffusion in the $N$-vortex problem on the plane. However, in the technique of [46], there is no such restriction on applicable systems, and instead we just assume that there is a saddle-center equilibrium with a homo- or heteroclinic connection on an invariant plane. In addition, unlike the other Melnikov-type techniques, we do not have to compute an infinite integral but only have to estimate a limit of a function in order to check a criterion for the occurrence of chaos. Owing to these advantages, the method is comparatively easily implemented by numerical means and actually made good use of in the present article. We also remark that another approach used in $[12,21,29]$ may also be numerically applied to the reduced system for $N=6$ although a symplectic transformation is further needed and the required numerical computation is very subtle since we have to determine whether the linearized flow along the homoclinic orbit to the saddle-center is rotational or not.

On the other hand, Champneys and Lord [6] presented a numerical technique to compute homoclinic orbits to periodic orbits in Hamiltonian systems and implemented it for a two-degree-of-freedom system via "AUTO". The technique has been recently extended to homo- and heteroclinic orbits to "general" nonhyperbolic trajectories in [50] and to stable and unstable manifolds of normally hyperbolic invariant manifolds in [51]. We apply the numerical technique of [51] to compute the stable and unstable manifolds of periodic orbits near saddle-centers for $N=5$ and 6 .

The paper consists of seven sections. In the next section, we derive twodegree-of-freedom reduced systems from the $N$-vortex problem by means of the reduction method proposed by Sakajo $[39,40]$. The reduction is successful when the number of the point vortices has the factor either 5 or 6 , i.e. $N=5 n$ or $N=6 n$ with $n \in \mathbb{N}$. In $\S 3$ we reformulate the reduced systems by introducing some symplectic transformations so that our numerical techniques given in $\S 4$ are applicable. In $\S 4$ we review the Melnikov-type technique of [46] and describe 
our numerical methods to detect transverse homo- or heteroclinic orbits to periodic orbits near saddle-centers. In $\S 5$ and $\S 6$, we apply these methods to the reduced systems for $N=6$ and $N=5$ respectively, and show the existence of chaotic orbits with the numerical means. The final section gives a summary and some discussions.

\section{Four-dimensional systems in the $N$-vortex prob- lem}

We reduce the $2 N$-dimensional system of (1.1) and (1.2) to four-dimensional systems following Ref. [39]. First, we review the results of the linear stability analysis of the $N$-ring $[37,38]$. The $N$-ring configuration at the line of latitude $\theta_{0}$ is represented by

$$
\Theta_{m}=\theta_{0}, \quad \Psi_{m}=\frac{2 \pi m}{N}, \quad m=1, \ldots, N .
$$

It is a relative equilibrium in (1.1) and (1.2) with the constant longitudinal velocity $V_{0}(N)$,

$$
V_{0}(N)=\frac{\Gamma_{1}-\Gamma_{2}}{4 \pi \sin ^{2} \theta_{0}}+\frac{\left(\Gamma_{1}+\Gamma_{2}+2 \pi\right) \cos \theta_{0}}{4 \pi \sin ^{2} \theta_{0}}-\frac{1}{2 N} \frac{\cos \theta_{0}}{\sin ^{2} \theta_{0}} .
$$

When we perturb the $N$-ring slightly,

$$
\Theta_{m}(t)=\theta_{0}+\varepsilon \theta_{m}(t), \quad \Psi_{m}(t)=\frac{2 \pi m}{N}+V_{0}(N) t+\varepsilon \varphi_{m}(t), \quad|\varepsilon| \ll 1,
$$

the linearized equations for $\left(\theta_{m}, \varphi_{m}\right)$ are given by

$$
\begin{aligned}
\dot{\theta}_{m} & =\frac{1}{4 \pi \sin \theta_{0}} \sum_{j \neq m}^{N} \frac{\varphi_{m}-\varphi_{j}}{1-\cos \frac{2 \pi}{N}(m-j)} \\
\dot{\varphi}_{m} & =\frac{1}{4 \pi \sin ^{3} \theta_{0}} \sum_{j \neq m}^{N} \frac{\theta_{m}-\theta_{j}}{1-\cos \frac{2 \pi}{N}(m-j)}+B_{N} \theta_{m}
\end{aligned}
$$

where $B_{N}$ denotes

$$
B_{N}=-\frac{\left(\Gamma_{1}+\Gamma_{2}+N-1\right)\left(1+\cos ^{2} \theta_{0}\right)+2\left(\Gamma_{1}-\Gamma_{2}\right) \cos \theta_{0}}{4 \pi \sin ^{3} \theta_{0}} .
$$

Eigenvalues of the linearized equations are represented by $\lambda_{m}^{ \pm}= \pm \sqrt{\xi_{m} \eta_{m}}$ for $m=0, \ldots, N-1$, in which

$$
\xi_{m}=\frac{m(N-m)}{4 \pi \sin \theta_{0}}, \quad \eta_{m}=\frac{m(N-m)}{4 \pi \sin ^{3} \theta_{0}}+B_{N}
$$

Thus we have $\lambda_{m}^{ \pm}=\lambda_{N-m}^{ \pm}$for $m=1, \ldots, N-1$ and $\lambda_{0}^{ \pm}=0$. Accordingly, the eigenvalues $\lambda_{M}^{ \pm}$are simple and $\lambda_{m}^{ \pm}$for $m=1, \ldots, M-1$ are double when $N=2 M$, while all the eigenvalues $\lambda_{m}^{ \pm}$for $m=1, \ldots, M$ are double when 
$N=2 M+1$. Furthermore, since the eigenvalues satisfy $\left(\lambda_{i}^{ \pm}\right)^{2}<\left(\lambda_{j}^{ \pm}\right)^{2}$ for $1 \leq i<j \leq M$, if $\left(\lambda_{k}^{ \pm}\right)^{2}<0<\left(\lambda_{k+1}^{ \pm}\right)^{2}$ holds for some $k \in \mathbb{N}$, then the eigenvalues $\lambda_{m}^{ \pm}$are neutrally stable for $m \leq k$, while $\lambda_{m}^{+}$is unstable for $m>k$. Thus, in other words, the stability of the $N$-ring agrees with that of the largest eigenvalues $\lambda_{M}^{ \pm}$.

Let the vectors $\boldsymbol{\psi}_{m}^{ \pm}$and $\boldsymbol{\phi}_{m}^{ \pm}$be defined by

$$
\begin{gathered}
\boldsymbol{\psi}_{m}^{ \pm}=\left(\sqrt{\xi_{m}}, \sqrt{\xi_{m}} \cos \frac{2 \pi}{N} m, \cdots, \sqrt{\xi_{m}} \cos \frac{2 \pi}{N}(N-1) m,\right. \\
\left. \pm \sqrt{\eta_{m}}, \pm \sqrt{\eta_{m}} \cos \frac{2 \pi}{N} m, \cdots, \pm \sqrt{\eta_{m}} \cos \frac{2 \pi}{N}(N-1) m\right)^{\mathrm{T}}, \\
\boldsymbol{\phi}_{m}^{ \pm}=\left(0, \sqrt{\xi_{m}} \sin \frac{2 \pi}{N} m, \cdots, \sqrt{\xi_{m}} \sin \frac{2 \pi}{N}(N-1) m,\right. \\
\left.0, \pm \sqrt{\eta_{m}} \sin \frac{2 \pi}{N} m, \cdots, \pm \sqrt{\eta_{m}} \sin \frac{2 \pi}{N}(N-1) m\right)^{\mathrm{T}},
\end{gathered}
$$

for $m=1, \ldots, M=[N / 2]$, in which $[x]$ symbolizes the largest integer less than or equal to $x$, and the superscript " $\mathrm{T}$ " represents the transpose operator. They are the eigenvectors corresponding to the double eigenvalues $\lambda_{m}^{ \pm}$for $m=$ $1, \ldots, M-1$. As for the eigenvalues $\lambda_{M}^{ \pm}, \boldsymbol{\psi}_{M}^{ \pm}$are the corresponding eigenvectors for $N=2 M$, while $\boldsymbol{\psi}_{M}^{ \pm}$and $\phi_{M}^{ \pm}$are those for $N=2 M+1$. Note that the number of the vectors $\boldsymbol{\psi}_{m}^{ \pm}$and $\phi_{m}^{ \pm}$are $2 N-2$. As described in [4], when the unstable $N$-ring is perturbed, the point vortices evolve in a bounded region away from the poles. So we blow up the phase space $\mathbb{P}_{N}$ at the poles of the sphere and identify it with $\mathbb{R}^{2 N}$. Let $\boldsymbol{\zeta}^{ \pm}=(1, \cdots, 1, \pm 1, \cdots, \pm 1)^{\mathrm{T}}$, which are orthogonal to $\boldsymbol{\psi}_{m}^{ \pm}$and $\boldsymbol{\phi}_{m}^{ \pm}$. The $2 N$ vectors $\boldsymbol{\psi}_{m}^{ \pm}, \boldsymbol{\phi}_{m}^{ \pm}$and $\boldsymbol{\zeta}^{ \pm}$express the evolution of the $N$ point vortices as a linear basis of the $2 N$-dimensional phase space $\mathbb{P}_{N}$ without the poles.

Next, we show how to obtain low-dimensional dynamical systems from (1.1) and (1.2). For a point $\left(\Theta_{1}, \cdots, \Theta_{N}, \Psi_{1}, \cdots, \Psi_{N}\right) \in \mathbb{P}_{N}$, we define the following two discrete transformations. The first one is the rotation of the $N$ point vortices around the $z$-axis by the angle $2 \pi p / N$, which we call the $p$-shift transformation $\sigma_{p}$ :

$$
\begin{aligned}
& \sigma_{p}: \quad \Theta_{m}^{\prime}=\Theta_{N-p+m}, \quad \Psi_{m}^{\prime}=\Psi_{N-p+m}+\frac{2 \pi p}{N} \quad \text { for } m=1, \ldots, p ; \\
& \Theta_{m}^{\prime}=\Theta_{m-p}, \quad \Psi_{m}^{\prime}=\Psi_{m-p}+\frac{2 \pi p}{N} \quad \text { for } m=p+1, \ldots, N \text {. }
\end{aligned}
$$

The second one is the pole reversal transformation, which replaces the north pole and the south pole by rotating them around the $x$-axis by the angle $\pi$. The pole reversal transformation for $N=2 M+1, \pi_{\mathrm{o}}:\left(\Theta_{m}, \Psi_{m}\right) \mapsto\left(\Theta_{m}^{\prime}, \Psi_{m}^{\prime}\right)$, is defined by

$$
\begin{array}{ll}
\Theta_{1}^{\prime}=\pi-\Theta_{1}, & \Psi_{1}^{\prime}=\Psi_{1}, \\
\Theta_{m}^{\prime}=\pi-\Theta_{N-m+2}, & \Psi_{m}^{\prime}=2 \Psi_{1}-\Psi_{N-m+2} \quad \text { for } m \neq 1 .
\end{array}
$$


Similarly, the pole reversal transformation for $N=2 M, \pi_{\mathrm{e}}:\left(\Theta_{m}, \Psi_{m}\right) \mapsto$ $\left(\Theta_{m}^{\prime}, \Psi_{m}^{\prime}\right)$, becomes

$$
\begin{array}{ll}
\Theta_{1}^{\prime}=\pi-\Theta_{1}, & \Psi_{1}^{\prime}=\Psi_{1}, \\
\Theta_{m}^{\prime}=\pi-\Theta_{N-m+2}, & \Psi_{m}^{\prime}=2 \pi+2 \Psi_{1}-\Psi_{N-m+2}
\end{array} \quad \text { for } m \neq 1 .
$$

With these discrete transformations we have the existence of the reduced dynamical systems [39].

Proposition 2.1. Let $N=p q,(p, q \in \mathbb{N})$. If $\sigma_{p} \boldsymbol{x}(0)=\boldsymbol{x}(0)$ for $\boldsymbol{X} \in \mathbb{P}_{N}$, then $\sigma_{p} \boldsymbol{X}(t)=\boldsymbol{X}(t)$ for $t \geq 0$. Furthermore, the $2 p$-dimensional linear subspace of $\mathbb{P}_{N}$,

$\boldsymbol{X}=\boldsymbol{X}_{\theta_{0}}+\sum_{k}\left(a_{k}^{+} \boldsymbol{\psi}_{k q}^{+}+a_{k}^{-} \boldsymbol{\psi}_{k q}^{-}+b_{k}^{+} \boldsymbol{\phi}_{k q}^{+}+b_{k}^{-} \boldsymbol{\phi}_{k q}^{-}\right)+c^{+} \boldsymbol{\zeta}^{+}+c^{-} \boldsymbol{\zeta}^{-}, \quad a_{k}^{ \pm}, b_{k}^{ \pm}, c^{ \pm} \in \mathbb{R}$,

is invariant with respect to $\sigma_{p}$, where $\boldsymbol{X}_{\theta_{0}}=\left(\theta_{0}, \theta_{0}, \ldots, \theta_{0}, 0, \frac{2 \pi}{N}, \ldots, \frac{2 \pi}{N}(N-1)\right)$.

The proposition claims that there exists a $2 p$-dimensional dynamical system for $N=p q$, which is also symbolized by $\mathbb{P}_{N}\left(\sigma_{p}\right)$. The $\sigma_{p}$ invariant orbit in $\mathbb{P}_{N}\left(\sigma_{p}\right)$ satisfies the following relations:

$$
\Theta_{k p+m}(t)=\Theta_{m}(t), \quad \Psi_{k p+m}(t)=\Psi_{m}(t)+\frac{2 \pi}{q} k
$$

for $k=0, \ldots, q-1$ and $m=1, \ldots, p$. Note that the reduced system can be obtained for every factor $p$ of $N$, the $N$-ring at any latitude $\theta_{0}$ and any strengths of the pole vortices.

Regarding the pole reversal transformations $\pi_{\mathrm{e}}$ and $\pi_{\mathrm{o}}$, we also have the following invariant dynamical systems.

Proposition 2.2. Let $N=2 M+1$ and $\Gamma_{1}=\Gamma_{2}$. Then, if $\pi_{\mathrm{o}} \boldsymbol{X}(0)=\boldsymbol{X}(0)$ for $\boldsymbol{X} \in \mathbb{P}_{N}$, then $\pi_{\mathrm{o}} \boldsymbol{X}(t)=\boldsymbol{X}(t)$ for $t \geq 0$. Furthermore, the $2 M$-dimensional linear subspace of $\mathbb{P}_{N}$,

$$
\boldsymbol{X}=\boldsymbol{X}_{\mathrm{o}}+\sum_{k=1}^{M}\left(b_{k}^{+} \boldsymbol{\phi}_{k}^{+}+b_{k}^{-} \boldsymbol{\phi}_{k}^{-}\right), \quad b_{k}^{ \pm} \in \mathbb{R},
$$

is invariant with respect to $\pi_{\mathrm{o}}$, where

$$
\boldsymbol{X}_{\mathrm{o}}=\left(\frac{\pi}{2}, \ldots, \frac{\pi}{2}, 0, \frac{2 \pi}{N}, \ldots, \frac{2 \pi M}{N},-\frac{2 \pi M}{N}, \ldots,-\frac{2 \pi}{N}\right) .
$$

Proposition 2.3. Let $N=2 M$ and $\Gamma_{1}=\Gamma_{2}$. Then, if $\pi_{\mathrm{e}} \boldsymbol{X}(0)=\boldsymbol{X}(0)$ for $\boldsymbol{X} \in \mathbb{P}_{N}$, then $\pi_{\mathrm{e}} \boldsymbol{X}(t)=\boldsymbol{X}(t)$ for $t \geq 0$. Furthermore, the $2(M-1)$ dimensional linear subspace of $\mathbb{P}_{N}$,

$$
\boldsymbol{X}=\boldsymbol{X}_{\mathrm{e}}+\sum_{k=1}^{M-1}\left(b_{k}^{+} \boldsymbol{\phi}_{k}^{+}+b_{k}^{-} \boldsymbol{\phi}_{k}^{-}\right), \quad b_{k}^{ \pm} \in \mathbb{R},
$$

is invariant with respect to $\pi_{\mathrm{e}}$, where $\boldsymbol{X}_{\mathrm{e}}=\left(\frac{\pi}{2}, \frac{\pi}{2}, \ldots, \frac{\pi}{2}, 0, \frac{2 \pi}{N}, \ldots, \frac{2 \pi}{N}(N-1)\right)$. 
The $\pi_{\mathrm{e}}$ and $\pi_{\mathrm{o}}$ invariant dynamical systems are also denoted by $\mathbb{P}_{N}\left(\pi_{\mathrm{e}}\right)$ and $\mathbb{P}_{N}\left(\pi_{\mathrm{o}}\right)$ respectively. Unlike $\mathbb{P}_{N}\left(\sigma_{p}\right)$, they exist under the additional assumption that the strengths of the pole vortices are identical. Furthermore, the invariant linear spaces (2.12) and (2.13) can be defined only for the $N$-ring at the equator and their dimensions are different. The $\pi_{\mathrm{o}}$ invariant orbit satisfies the following relations,

$$
\Theta_{1}=\frac{\pi}{2}, \quad \Psi_{1}=0, \quad \Theta_{m}+\Theta_{N-m+2}=\pi, \quad \Psi_{m}+\Psi_{N-m+2}=0 \quad \text { for } m \neq 1
$$

Similarly, the $\pi_{\mathrm{e}}$ invariant orbit satisfies

$$
\Theta_{1}=\frac{\pi}{2}, \quad \Psi_{1}=0, \quad \Theta_{m}+\Theta_{N-m+2}=\pi, \quad \Psi_{m}+\Psi_{N-m+2}=2 \pi \quad \text { for } m \neq 1 .
$$

As a matter of fact, these relations are equivalent owing to $\Psi_{m} \in(\mathbb{R} / 2 \pi \mathbb{Z})$.

Combining the $p$-shift and the pole reversal transformations, we have more propositions, which are used to obtain four-dimensional systems in the paper.

Proposition 2.4. Let $N=2 M=p q,(p, q \in \mathbb{N})$ and $\Gamma_{1}=\Gamma_{2}$. Then, if $\sigma_{p} \pi_{\mathrm{e}} \boldsymbol{X}(0)=\boldsymbol{X}(0)$ for $\boldsymbol{X} \in \mathbb{P}_{N}$, then $\sigma_{p} \pi_{\mathrm{e}} \boldsymbol{X}(t)=\boldsymbol{X}(t)$ for $t \geq 0$. Furthermore, the set of

$$
\boldsymbol{X}=\boldsymbol{X}_{\mathrm{e}}+\sum_{k}\left(b_{k}^{+} \boldsymbol{\phi}_{k q}^{+}+b_{k}^{-} \boldsymbol{\phi}_{k q}^{-}\right), \quad b_{k}^{ \pm} \in \mathbb{R},
$$

is invariant with respect to $\sigma_{p} \pi_{\mathrm{e}}$, where $\boldsymbol{X}_{\mathrm{e}}$ represents the $N$-ring at the equator.

Proposition 2.5. Let $N=2 M+1=p q,(p, q \in \mathbb{N})$ and $\Gamma_{1}=\Gamma_{2}$. Then, if $\sigma_{p} \pi_{\mathrm{o}} \boldsymbol{X}(0)=\boldsymbol{X}(0)$ for $\boldsymbol{X} \in \mathbb{P}_{N}$, then $\sigma_{p} \pi_{\mathrm{o}} \boldsymbol{X}(t)=\boldsymbol{X}(t)$ for $t \geq 0$. Furthermore, the set of

$$
\boldsymbol{X}=\boldsymbol{X}_{\mathrm{o}}+\sum_{k}\left(b_{k}^{+} \boldsymbol{\phi}_{k q}^{+}+b_{k}^{-} \boldsymbol{\phi}_{k q}^{-}\right), \quad b_{k}^{ \pm} \in \mathbb{R},
$$

is invariant with respect to $\sigma_{p} \pi_{\mathrm{o}}$, where $\boldsymbol{X}_{\mathrm{o}}$ represents the $N$-ring at the equator.

Now, we derive low-dimensional dynamical systems with the above propositions. For the simplest case, where the dimension of the system is two, it is sufficient to plot the contour lines of the reduced Hamiltonian to investigate the evolution of the unstable $N$-ring, since it is integrable. The second simplest case is a four-dimensional dynamical system with two degrees of freedom, which is no longer integrable. Then, the unstable $N$-ring possibly exhibits a complex behavior. Among the $p$-shift invariant systems, it is $\mathbb{P}_{N}\left(\sigma_{2}\right)$ whose dimension is four, but it is integrable because of the existence of the invariant quantity $I$. Indeed, $\mathbb{P}_{N}\left(\sigma_{2}\right)$ has already been investigated by the first author [37], which showed the transition of the global structure of periodic orbits. Regarding $\mathbb{P}_{N}\left(\pi_{\mathrm{o}}\right)$ and $\mathbb{P}_{N}\left(\pi_{\mathrm{e}}\right)$, the four dimensional reduced system exists for $N=5$ and $N=6$. They are not integrable, since the invariant $I$ is always zero due to (2.14) and (2.15). 
Moreover, the reduced systems $\mathbb{P}_{N}\left(\sigma_{p} \pi_{\mathrm{o}}\right)$ and $\mathbb{P}_{N}\left(\sigma_{p} \pi_{\mathrm{e}}\right)$ become four-dimensional only when $N=5 n$ or $N=6 n$, which we are going to confirm in what follows. Suppose that a system is $\sigma_{p} \pi_{\mathrm{e}}$-invariant. Then, for $N=2 M$, it follows from Proposition 2.3 that the $\pi_{\mathrm{e}}$ invariant space (2.13) is spanned by $\phi_{k}^{ \pm}$for $k=1, \ldots, M-1$. In addition, $N$ should have the factor $p$, i.e. $N=p n$ for $n>1$. Then the dimension of the $\sigma_{p} \pi_{\mathrm{e}}$ invariant system is four, when

$$
\left[\frac{M-1}{n}\right]=\left[\frac{1}{n}\left(\frac{p n}{2}-1\right)\right]=\left[\frac{p}{2}-\frac{1}{n}\right]=2 .
$$

Since $\left[\frac{p}{2}-\frac{1}{n}\right] \geq 3$ for $p \geq 7$ and less than 2 for $p \leq 4$, we have $p=5$ and 6 . In the similar manner, only $p=5$ is allowed for $N=2 M+1$.

Thus the phase spaces of the two-degree-of-freedom reduced dynamical systems embedded in the $N$ vortex problem are $\mathbb{P}_{5}\left(\pi_{\mathrm{o}}\right), \mathbb{P}_{6}\left(\pi_{\mathrm{e}}\right), \mathbb{P}_{N}\left(\sigma_{5} \pi_{\mathrm{e}}\right)$, $\mathbb{P}_{N}\left(\sigma_{5} \pi_{\mathrm{o}}\right)$ and $\mathbb{P}_{N}\left(\sigma_{6} \pi_{\mathrm{e}}\right)$. According to Propositions 2.4 and 2.5, the linear subspaces of the reduced systems are expressed by the set of

$$
\boldsymbol{X}=\boldsymbol{X}_{\mathrm{o}, \mathrm{e}}+b_{1}^{+} \boldsymbol{\phi}_{n}^{+}+b_{1}^{-} \boldsymbol{\phi}_{n}^{-}+b_{2}^{+} \boldsymbol{\phi}_{2 n}^{+}+b_{2}^{-} \boldsymbol{\phi}_{2 n}^{-},
$$

which is the eigenspace corresponding to the eigenvalues $\lambda_{n}^{ \pm}$and $\lambda_{2 n}^{ \pm}$. Hence, if $\left(\lambda_{n}^{ \pm}\right)^{2}<0<\left(\lambda_{2 n}^{ \pm}\right)^{2}$, the $N$-ring at the equator becomes a saddle-center equilibrium in the four-dimensional system. Furthermore, the reduced invariant system $\mathbb{P}_{N}\left(\sigma_{6} \pi_{\mathrm{e}}\right)$ contains the two-dimensional $\sigma_{3} \pi_{\mathrm{e}}$ invariant system $\mathbb{P}_{N}\left(\sigma_{3} \pi_{\mathrm{e}}\right)$ due to $N=6 n=3 \times(2 n)$, for which the coordinates in the phase space are represented by

$$
\boldsymbol{X}=\boldsymbol{X}_{\mathrm{e}}+b_{2}^{+} \boldsymbol{\phi}_{2 n}^{+}+b_{2}^{-} \boldsymbol{\phi}_{2 n}^{-} .
$$

Since the two-dimensional dynamical system is integrable, a contour curve of the reduced Hamiltonian corresponds to an orbit of the restricted $N$ point vortices. Furthermore, since $\phi_{2 n}^{ \pm}$span the eigenspace of $\lambda_{2 n}^{ \pm}$, the unstable and stable manifolds lie in the phase space (2.18). Thus the reduced system acquires the situation where the abstract theory of two-degree-of-freedom Hamiltonian with saddle-centers [46] is applicable. The two-dimensional system plays a significant role in the application of the Melnikov analysis to Hamiltonians with the saddlecenters for $\mathbb{P}_{N}\left(\sigma_{6} \pi_{\mathrm{e}}\right)$.

We finally note that there exist unstable directions normal to the invariant four-dimensional space in the full phase space $\mathbb{P}_{N}$ since $\lambda_{m}^{+}$is a positive real for $2 n<m \leq 3 n$. Thus, the invariant spaces are unstable and all orbits on it are unstable. See also $\S 7$ for more details when $N=6$.

\section{Symplectic Formulations}

\subsection{Equations for $\mathbb{P}_{N}\left(\sigma_{6} \pi_{\mathrm{e}}\right)$}

We reformulate the four-dimensional dynamical systems $\mathbb{P}_{N}\left(\sigma_{6} \pi_{\mathrm{e}}\right)$. It follows from (2.11) and (2.15) that we have the relations for the $\sigma_{6} \pi_{\mathrm{e}}$ invariant orbit, which are represented by

$$
\begin{gathered}
\Theta_{1}=\Theta_{6 k-5}=\frac{\pi}{2}, \quad \Theta_{2}=\Theta_{6 k-4}, \quad \Theta_{3}=\Theta_{6 k-3}, \quad \Theta_{4}=\Theta_{6 k-2}=\frac{\pi}{2}, \\
\Theta_{5}=\Theta_{6 k-1}, \quad \Theta_{6}=\Theta_{6 k}, \quad \Theta_{2}+\Theta_{6}=\pi, \quad \Theta_{3}+\Theta_{5}=\pi,
\end{gathered}
$$


and

$$
\begin{gathered}
\Psi_{6 k-5}=\frac{2 \pi}{n}(k-1), \quad \Psi_{6 k-4}=\Psi_{2}+\frac{2 \pi}{n}(k-1), \quad \Psi_{6 k-3}=\Psi_{3}+\frac{2 \pi}{n}(k-1), \\
\Psi_{6 k-2}=\frac{\pi}{n}+\frac{2 \pi}{n}(k-1), \quad \Psi_{6 k-1}=\Psi_{5}+\frac{2 \pi}{n}(k-1), \quad \Psi_{6 k}=\Psi_{6}+\frac{2 \pi}{n}(k-1), \\
\Psi_{2}+\Psi_{6}=\frac{2 \pi}{n}, \quad \Psi_{3}+\Psi_{5}=\frac{2 \pi}{n},
\end{gathered}
$$

for $k=1, \ldots, n$.

Note that Equations (1.1) and (1.2) are written in a symplectic form by using the variables $p_{m}=\cos \Theta_{m}$ and $q_{m}=\Psi_{m}$ as follows [30].

$$
\dot{q}_{m}=\frac{\partial H}{\partial p_{m}}, \quad \dot{p}_{m}=-\frac{\partial H}{\partial q_{m}}, \quad m=1, \ldots, N .
$$

Introducing the following generating function $W\left(P_{m}, q_{m}\right)$,

$$
W\left(P_{m}, q_{m}\right)=P_{1} q_{1}+\sum_{m=2}^{N} P_{m}\left(q_{m}-q_{m-1}\right),
$$

we define the symplectic transformation $\left(q_{m}, p_{m}\right) \mapsto\left(Q_{m}, P_{m}\right)$ by

$$
\begin{aligned}
& Q_{1}=q_{1}, \quad Q_{m}=\frac{\partial W}{\partial P_{m}}=q_{m}-q_{m-1} \quad \text { for } m=2, \ldots, N, \\
& p_{N}=P_{N}, \quad p_{m}=\frac{\partial W}{\partial q_{m}}=P_{m}-P_{m+1} \quad \text { for } m=1, \ldots, N-1 .
\end{aligned}
$$

With the new variables $\left(Q_{m}, P_{m}\right)$, we rewrite $(3.3)$ for the $\sigma_{6} \pi_{\mathrm{e}}$ invariant dynamical systems.

First, the relations $(3.1)$ are restated in terms of $p_{m}$. For $k=1, \ldots n$, they are equivalent to

$$
\begin{gathered}
p_{6 k-5}=p_{6 k-2}=0, \quad p_{6 k-4}=p_{2}, \quad p_{6 k-3}=p_{3}, \quad p_{6 k-1}=p_{5}, \quad p_{6 k}=p_{6}, \\
p_{6 k-4}+p_{6 k}=0, \quad p_{6 k-3}+p_{6 k-1}=0 .
\end{gathered}
$$

With the new variables $P_{m}, p_{6 k-5}=p_{6 k-2}=0$ yields

$$
P_{6 k-5}=P_{6 k-4}, \quad P_{6 k-2}=P_{6 k-1},
$$

for $k=1, \ldots, n$. It follows from $p_{6 k-3}+p_{6 k-1}=0$ and (3.7) that we have

$$
P_{6 k-3}=P_{6 k},
$$

for $k \leq n$. Noting that $p_{6 k-3}+p_{6 k-1}=p_{6 k+3}+p_{6 k-1}=0$, we also have

$$
P_{6 k+3}-P_{6 k+4}+P_{6 k-1}-P_{6 k}=0, \quad \text { for } k<n .
$$

On the other hand, $p_{6 k+4}+p_{6 k}=0$ implies that $P_{6 k-4}-P_{6 k-3}+P_{6 k}-P_{6 k+1}=0$ for $k<n$, which is reduced to $P_{6 k-4}=P_{6 k+1}$ due to (3.8). For $k=n$, since $p_{6 n}=P_{6 n}$, we have $P_{6 n-4}=0$. Hence, we have

$$
P_{1}=P_{2}=P_{7}=\cdots=P_{6 k-5}=P_{6 k-4}=P_{6 k+1}=\cdots=P_{6 n-4}=0 .
$$


Furthermore, $p_{6 k-4}+p_{6 k}=p_{6 k+2}+p_{6 k}=0$ is equivalent to $P_{6 k+2}-P_{6 k+3}+$ $P_{6 k}-P_{6 k+1}=0$, which leads to $P_{6 k}=P_{6 k+3}$ due to (3.10). Substituting it into (3.9), we obtain $P_{6 k+4}=P_{6 k-1}$. Consequently, we have the following relations of $P_{m}$ for the $\sigma_{6} \pi_{\mathrm{e}}$ invariant orbit.

$$
\begin{aligned}
& P_{1}=P_{2}=P_{7}=\cdots=P_{6 k-5}=P_{6 k-4}=P_{6 k+1}=\cdots=P_{6 n-4}=0 \\
& P_{3}=P_{6}=P_{9}=\cdots=P_{6 k-3}=P_{6 k}=P_{6 k+3}=\cdots=P_{6 n-3}=P_{6 n} \\
& P_{4}=P_{5}=P_{10}=\cdots=P_{6 k-2}=P_{6 k-1}=P_{6 k+4}=\cdots=P_{6 n-2}=P_{6 n-1}
\end{aligned} .
$$

Next, we rewrite the relation (3.2) in terms of $Q_{m}$. It follows from $\Psi_{6 k-5}=$ $\frac{2 \pi}{n}(k-1)$ and $\Psi_{6 k-4}=\Psi_{2}+\frac{2 \pi}{n}(k-1)$ that we obtain $Q_{6 k-4}=\Psi_{6 k-5}-\Psi_{6 k-4}=$ $\Psi_{2}=Q_{2}$. Similarly, we have $Q_{6 k-3}=Q_{3}, Q_{6 k-2}=Q_{4}, Q_{6 k-1}=Q_{5}$ and $Q_{6 k}=$ $Q_{6}$. The relations $\Psi_{2}+\Psi_{6}=\frac{2 \pi}{n}$ and $\Psi_{3}+\Psi_{5}=\frac{2 \pi}{n}$ yield $\Psi_{3}-\Psi_{2}+\Psi_{5}-\Psi_{6}=0$, equivalently

$$
Q_{3}=Q_{6} .
$$

Moreover, the other relations $\Psi_{4}=\frac{\pi}{n}$ and $\Psi_{2}+\Psi_{6}=\frac{2 \pi}{n}$ are represented by

$$
Q_{1}+Q_{2}+Q_{3}+Q_{4}=\frac{\pi}{n}, \quad 2 Q_{1}+2 Q_{2}+Q_{3}+Q_{4}+Q_{5}+Q_{6}=\frac{2 \pi}{n} .
$$

Hence, it follows from (3.12) and (3.13) that $Q_{4}=Q_{5}$. Consequently, we have the relations of the $\sigma_{6} \pi_{\mathrm{e}}$ invariant orbit for $Q_{m}$.

$$
\begin{aligned}
& Q_{1}=0, \quad Q_{2}+Q_{3}+Q_{4}=\frac{\pi}{n}, \\
& Q_{3}=Q_{6}=\cdots=Q_{6 k-3}=Q_{6 k}=\cdots=Q_{6 n-3}=Q_{6 n} \\
& Q_{4}=Q_{5}=\cdots=Q_{6 k-2}=Q_{6 k-1}=\cdots=Q_{6 n-2}=Q_{6 n-1} .
\end{aligned}
$$

The Hamiltonian reduced by the $\sigma_{6}$ invariance with the identical pole vortex points, say $\Gamma_{1}=\Gamma_{2} \equiv \Gamma$, is given as follows [39].

$$
\begin{aligned}
H= & -\frac{n}{4 \pi} \sum_{m=1}^{6} \sum_{m<j}^{6} \sum_{l=0}^{n-1} \log \left(1-\cos \Theta_{m} \cos \Theta_{j}-\sin \Theta_{m} \sin \Theta_{j} \cos \left(\Psi_{m}-\Psi_{j}+\frac{2 \pi}{n} l\right)\right) \\
& -\frac{n(n-1)+n \Gamma}{4 \pi} \sum_{m=1}^{6} \log \left(1-\cos ^{2} \Theta_{m}\right) .
\end{aligned}
$$

Substituting $\cos \Theta_{m}=p_{m}=P_{m}-P_{m+1}, \sin \Theta_{m}=\sqrt{1-p_{m}^{2}}=\sqrt{1-\left(P_{m}-P_{m+1}\right)^{2}}$ and the relations (3.11) and (3.14) into the above Hamiltonian, we obtain the 
Hamiltonian $H_{n}^{(6)}$ for $\mathbb{P}_{N}\left(\sigma_{6} \pi_{\mathrm{e}}\right)$ with two-degree-of-freedom, which is

$$
\begin{aligned}
H_{n}^{(6)} & \left(Q_{3}, Q_{4}, P_{3}, P_{4}\right) \\
= & -\frac{n}{2 \pi} \sum_{l=0}^{n-1} \log \left(1-\sqrt{1-P_{3}^{2}} \cos \left(Q_{3}+Q_{4}+\frac{2 \pi}{n} l-\frac{\pi}{n}\right)\right) \\
& -\frac{n}{2 \pi} \sum_{l=0}^{n-1} \log \left(1-\sqrt{1-\left(P_{3}-P_{4}\right)^{2}} \cos \left(Q_{4}+\frac{2 \pi}{n} l-\frac{\pi}{n}\right)\right) \\
& -\frac{n}{2 \pi} \sum_{l=0}^{n-1} \log \left(1-\sqrt{1-P_{3}^{2}} \cos \left(Q_{3}+Q_{4}+\frac{2 \pi}{n} l\right)\right) \\
& -\frac{n}{2 \pi} \sum_{l=0}^{n-1} \log \left(1-\sqrt{1-\left(P_{3}-P_{4}\right)^{2}} \cos \left(Q_{4}+\frac{2 \pi}{n} l\right)\right) \\
& -\frac{n}{2 \pi} \sum_{l=0}^{n-1} \log \left(1+P_{3}\left(P_{3}-P_{4}\right)-\sqrt{\left(1-P_{3}^{2}\right)\left(1-\left(P_{3}-P_{4}\right)^{2}\right)} \cos \left(Q_{3}+\frac{2 \pi}{n} l\right)\right) \\
& -\frac{n}{2 \pi} \sum_{l=0}^{n-1} \log \left(1-P_{3}\left(P_{3}-P_{4}\right)-\sqrt{\left(1-P_{3}^{2}\right)\left(1-\left(P_{3}-P_{4}\right)^{2}\right)} \cos \left(Q_{3}+2 Q_{4}+\frac{2 \pi}{n} l\right)\right) \\
& -\frac{n}{4 \pi} \sum_{l=0}^{n-1} \log \left(1+P_{3}^{2}-\left(1-P_{3}^{2}\right) \cos \left(2 Q_{3}+2 Q_{4}+\frac{2 \pi}{n} l\right)\right) \\
& -\frac{n}{4 \pi} \sum_{l=0}^{n-1} \log \left(1+\left(P_{3}-P_{4}\right)^{2}-\left(1-\left(P_{3}-P_{4}\right)^{2}\right) \cos \left(2 Q_{4}+\frac{2 \pi}{n} l\right)\right) \\
- & \frac{n(n-1)+n \Gamma}{2 \pi} \log \left(1-P_{3}^{2}\right) \log \left(1-\left(P_{3}-P_{4}\right)^{2}\right) .
\end{aligned}
$$

Note that the Hamiltonian $H_{1}^{(6)}$ is identical to that for $\mathbb{P}_{6}\left(\pi_{\mathrm{e}}\right)$. As we explained in the previous section, $\mathbb{P}_{N}\left(\sigma_{6} \pi_{\mathrm{e}}\right)$ contains the two-dimensional invariant system $\mathbb{P}_{N}\left(\sigma_{3} \pi_{\mathrm{e}}\right)$; in addition to (3.1) and (3.2), the $\sigma_{3} \pi_{\mathrm{e}}$ invariant orbit satisfies

$$
\Theta_{1}=\Theta_{4}, \quad \Theta_{2}=\Theta_{5}, \quad \Theta_{3}=\Theta_{6}, \quad \Theta_{2}+\Theta_{3}=\pi, \quad \Psi_{2}+\Psi_{3}=\frac{\pi}{n},
$$

which are equivalent to

$$
P_{2}=P_{4}=0, \quad Q_{2}=Q_{4}, \quad Q_{3}+2 Q_{4}=\frac{\pi}{n} .
$$

Hence, substituting (3.17) into (3.16), we have the reduced Hamiltonian with one-degree-of-freedom for the symplectic variables $\left(P_{3}, Q_{3}\right)$, say $H_{2 n}^{(3)}$. Since $\mathbb{P}_{N}\left(\sigma_{3} \pi_{\mathrm{e}}\right)$ is integrable, the contour line of $H_{2 n}^{(3)}$ represents the $\sigma_{3} \pi_{\mathrm{e}}$ invariant orbit. Remembering that the phase space of the two-dimensional integrable system is the eigenspace corresponding to the eigenvalues $\lambda_{2 n}^{ \pm}$in the saddle direction, we just plot the contour lines to obtain the unstable and the stable manifolds for the unstable $N$-ring. 


\subsection{Equations for $\mathbb{P}_{N}\left(\sigma_{5} \pi_{\mathrm{e}}\right)$ and $\mathbb{P}_{N}\left(\sigma_{5} \pi_{\mathrm{o}}\right)$}

When $N=5 n$, there exist the four-dimensional dynamical systems $\mathbb{P}_{N}\left(\sigma_{5} \pi_{\mathrm{e}}\right)$ for even $n$, and $\mathbb{P}_{N}\left(\sigma_{5} \pi_{\mathrm{o}}\right)$ for odd $n$. The two systems give the same Hamiltonian, since the relation of $\pi_{\mathrm{e}}$ invariant orbit (2.15) is equivalent to that of $\pi_{\mathrm{o}}$ invariant orbit $(2.14)$ due to $\Psi_{m} \in(\mathbb{R} / 2 \pi \mathbb{Z})$. The $\sigma_{5} \pi_{\mathrm{e}}$ and $\sigma_{5} \pi_{\mathrm{o}}$ invariant orbits satisfy the following relations for $k=1, \ldots, n$ :

$$
\begin{aligned}
& \Theta_{5 k-4}=\frac{\pi}{2}, \quad \Theta_{5 k-3}=\Theta_{2}, \quad \Theta_{5 k-2}=\Theta_{3}, \quad \Theta_{5 k-1}=\Theta_{4}, \quad \Theta_{5 k}=\Theta_{5}, \\
& \Theta_{2}+\Theta_{5}=\pi, \quad \Theta_{3}+\Theta_{4}=\pi
\end{aligned}
$$

and

$$
\begin{aligned}
& \Psi_{5 k-4}=\frac{2 \pi}{n}(k-1), \quad \Psi_{5 k-3}=\Psi_{2}+\frac{2 \pi}{n}(k-1), \quad \Psi_{5 k-2}=\Psi_{3}+\frac{2 \pi}{n}(k-1), \\
& \Psi_{5 k-1}=\Psi_{4}+\frac{2 \pi}{n}(k-1), \quad \Psi_{5 k}=\Psi_{5}+\frac{2 \pi}{n}(k-1), \\
& \Psi_{2}+\Psi_{5}=\frac{2 \pi}{n}, \quad \Psi_{3}+\Psi_{4}=\frac{2 \pi}{n} .
\end{aligned}
$$

With the same generating function $W\left(P_{m}, q_{m}\right)$, we define the symplectic transformation of variables $\left(q_{m}, p_{m}\right) \mapsto\left(Q_{m}, P_{m}\right)$. In the similar way in $N=6 n$, we rewrite the relations in terms of $\left(Q_{m}, P_{m}\right)$, which are given by

$$
\begin{aligned}
& Q_{3}=Q_{5}=\cdots=Q_{5 k-2}=Q_{5 k}=\cdots=Q_{5 n-2}=Q_{5 n}, \quad Q_{5 k-1}=Q_{4}, \\
& Q_{5 k-3}=Q_{2}, \quad Q_{5 k-4}=Q_{1}=0, \quad Q_{2}+Q_{3}+\frac{Q_{4}}{2}=\frac{\pi}{n}, \\
& P_{1}=P_{2}=P_{6}=\cdots=P_{5 k-4}=P_{5 k-3}=P_{5 k+1}=\cdots=P_{5 n-4}=P_{5 n-3}=0, \\
& P_{3}=P_{5}=P_{8}=\cdots=P_{5 k-2}=P_{5 k}=P_{5 k+3}=\cdots=P_{5 n-2}=P_{5 n}, \quad P_{4}=P_{5 k-1} .
\end{aligned}
$$


Substitution of the relation (3.18) into the $\sigma_{5}$ invariant Hamiltonian leads to the Hamiltonian $H_{n}^{(5)}$ for $\mathbb{P}_{N}\left(\sigma_{5} \pi_{\mathrm{e}}\right)$ and $\mathbb{P}_{N}\left(\sigma_{5} \pi_{\mathrm{o}}\right)$.

$$
\begin{aligned}
H_{n}^{(5)} & \left(Q_{3}, Q_{4}, P_{3}, P_{4}\right) \\
= & -\frac{n}{2 \pi} \sum_{l=0}^{n-1} \log \left(1-\sqrt{1-P_{3}^{2}} \cos \left(Q_{3}+\frac{Q_{4}}{2}+\frac{\pi}{n}+\frac{2 \pi}{n} l\right)\right) \\
& -\frac{n}{2 \pi} \sum_{l=0}^{n-1} \log \left(1-\sqrt{1-\left(P_{3}-P_{4}\right)^{2}} \cos \left(\frac{Q_{4}}{2}+\frac{\pi}{n}+\frac{2 \pi}{n} l\right)\right) \\
& -\frac{n}{2 \pi} \sum_{l=0}^{n-1} \log \left(1+P_{3}\left(P_{3}-P_{4}\right)-\sqrt{\left(1-P_{3}^{2}\right)\left(1-\left(P_{3}-P_{4}\right)^{2}\right)} \cos \left(Q_{3}+\frac{2 \pi}{n} l\right)\right) \\
& -\frac{n}{2 \pi} \sum_{l=0}^{n-1} \log \left(1-P_{3}\left(P_{3}-P_{4}\right)-\sqrt{\left(1-P_{3}^{2}\right)\left(1-\left(P_{3}-P_{4}\right)^{2}\right)} \cos \left(Q_{3}+Q_{4}+\frac{2 \pi}{n} l\right)\right) \\
& -\frac{n}{4 \pi} \sum_{l=0}^{n-1} \log \left(1+P_{3}^{2}-\left(1-P_{3}^{2}\right) \cos \left(2 Q_{3}+Q_{4}+\frac{2 \pi}{n} l\right)\right) \\
& -\frac{n}{4 \pi} \sum_{l=0}^{n-1} \log \left(1+\left(P_{3}-P_{4}\right)^{2}-\left(1-\left(P_{3}-P_{4}\right)^{2}\right) \cos \left(Q_{4}+\frac{2 \pi}{n} l\right)\right) \\
& -\frac{n(n-1)+n \Gamma}{2 \pi} \log \left(1-P_{3}^{2}\right)\left(1-\left(P_{3}-P_{4}\right)^{2}\right) .
\end{aligned}
$$

The Hamiltonian $H_{1}^{(5)}$ is equivalent to that for $\mathbb{P}_{5}\left(\pi_{\mathrm{o}}\right)$. Unlike $\mathbb{P}_{N}\left(\sigma_{6} \pi_{\mathrm{e}}\right)$, there is no two-dimensional invariant dynamical system embedded in $\mathbb{P}_{N}\left(\sigma_{5} \pi_{\mathrm{e}}\right)$ and $\mathbb{P}_{N}\left(\sigma_{5} \pi_{\mathrm{o}}\right)$, since the number " 5 " is prime. This indicates that it is not easy to obtain the unstable and stable manifolds in the saddle direction.

\section{Analytical and numerical techniques}

In this section we give our analytical and numerical techniques to show that there exist transverse homo- and heteroclinic orbits to periodic orbits near saddle-centers and consequently chaotic dynamics occurs.

\subsection{Melnikov-type method}

Our analytical technique is the Melnikov-type method of [46] and its extension for two-degree-of-freedom Hamiltonian systems with saddle-centers. We outline the method in our context.

We consider two-degree-of-freedom Hamiltonian systems of the form

$$
\dot{\boldsymbol{x}}=J_{1} \mathrm{D}_{x} H(\boldsymbol{x}, \boldsymbol{y}), \quad \dot{\boldsymbol{y}}=J_{1} \mathrm{D}_{y} H(\boldsymbol{x}, \boldsymbol{y}), \quad(\boldsymbol{x}, \boldsymbol{y}) \in \mathbb{R}^{2} \times \mathbb{R}^{2},
$$

where $H: \mathbb{R}^{2} \times \mathbb{R}^{2} \rightarrow \mathbb{R}$ is $C^{r+1}(r \geq 3)$ and $J_{1}$ is the $2 \times 2$ symplectic matrix,

$$
J_{1}=\left(\begin{array}{cc}
0 & 1 \\
-1 & 0
\end{array}\right)
$$


(a)

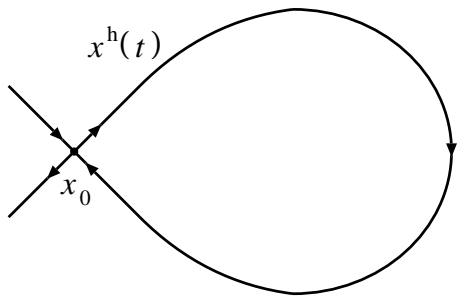

(b)

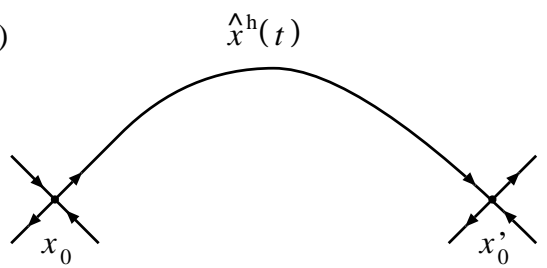

Figure 1: Phase portraits on the $\boldsymbol{x}$-plane: (a) a hyperbolic saddle and a homoclinic orbit; (b) two hyperbolic saddles and a heteroclinic orbit.

We first treat a case in which a saddle-center has a homoclinic orbit in (4.1). More precisely, we make the following assumptions on (4.1).

(A1) The $\boldsymbol{x}$-plane, $\left\{(\boldsymbol{x}, \boldsymbol{y}) \in \mathbb{R}^{2} \times \mathbb{R}^{2} \mid \boldsymbol{y}=\mathbf{0}\right\}$, is invariant under the flow of (4.1), i.e., $\mathrm{D}_{y} H(\boldsymbol{x}, \mathbf{0})=\mathbf{0}$ for any $\boldsymbol{x} \in \mathbb{R}^{2}$.

(A2) There is a saddle-center at $(\boldsymbol{x}, \boldsymbol{y})=\left(\boldsymbol{x}_{0}, \mathbf{0}\right)$ on the $\boldsymbol{x}$-plane such that the matrix $J_{1} \mathrm{D}_{x}^{2} H\left(\boldsymbol{x}_{0}, \mathbf{0}\right)$ has a pair of real eigenvalues $\pm \lambda$ and the matrix $J_{1} \mathrm{D}_{y}^{2} H\left(\boldsymbol{x}_{0}, \mathbf{0}\right)$ has a pair of purely imaginary eigenvalues $\pm i \omega(\lambda, \omega>0)$.

Assumption (A2) implies that there exists a one-parameter family of periodic orbits near the saddle-center $\left(\boldsymbol{x}_{0}, \mathbf{0}\right)$ by the Lyapunov center theorem [28]. In addition, the system restricted on the $\boldsymbol{x}$-plane,

$$
\dot{\boldsymbol{x}}=J_{1} \mathrm{D}_{x} H(\boldsymbol{x}, \mathbf{0}),
$$

also has a hyperbolic saddle at $\boldsymbol{x}=\boldsymbol{x}_{0}$. We also assume the following on (4.2).

(A3) The saddle $\boldsymbol{x}=\boldsymbol{x}_{0}$ of (4.2) has a homoclinic orbit $\boldsymbol{x}^{\mathrm{h}}(t)$. See Fig. 1(a).

Let $\Phi(t)$ and $\Psi(t)$ be fundamental matrices for the variational equations in the $\boldsymbol{y}$-direction about the saddle-center $\left(\boldsymbol{x}_{0}, \mathbf{0}\right)$ and homoclinic orbit $(\boldsymbol{x}, \boldsymbol{y})=$ $\left(\boldsymbol{x}^{\mathrm{h}}(t), \mathbf{0}\right)$, i.e.,

$$
\dot{\boldsymbol{\eta}}=J_{1} \mathrm{D}_{y}^{2} H\left(\boldsymbol{x}_{0}, \mathbf{0}\right) \boldsymbol{\eta}
$$

and

$$
\dot{\boldsymbol{\eta}}=J_{1} \mathrm{D}_{y}^{2} H\left(\boldsymbol{x}^{\mathrm{h}}(t), \mathbf{0}\right) \boldsymbol{\eta}
$$

respectively, such that $\Phi(0)=I_{2}$ with $I_{2}$ the $2 \times 2$ identity matrix. We call (4.4) the normal variational equation (NVE) along $\boldsymbol{x}^{\mathrm{h}}(t)$. Then the limits

$$
B_{ \pm}=\lim _{t \rightarrow \pm \infty} \Phi(-t) \Psi(t)
$$

exist (see Lemma 3.1 of [46] for the proof), and we set $B_{0}=B_{+} B_{-}^{-1}$. Define the Melnikov function $M\left(t_{0}\right)$ as

$$
M\left(t_{0}\right)=m\left(\boldsymbol{e}_{1}\right)-m\left(B_{0} \Phi\left(t_{0}\right) \boldsymbol{e}_{1}\right),
$$

where

$$
m(\boldsymbol{\eta})=\frac{1}{2} \boldsymbol{\eta} \cdot \mathrm{D}_{y}^{2} H\left(\boldsymbol{x}_{0}, \mathbf{0}\right) \boldsymbol{\eta},
$$


and "." represents the inner product $\boldsymbol{e}_{1}=(1,0)^{\mathrm{T}}$ with ${ }^{\mathrm{T}}$ the transpose operator. Under assumptions (A1)-(A3) we state the result of Theorem 1.1 of [46] as follows.

Theorem 4.1. Let $\gamma$ be a periodic orbit sufficiently close to the saddle-center $\left(\boldsymbol{x}_{0}, \mathbf{0}\right)$. Suppose that $M\left(t_{0}\right)$ has a simple zero, i.e., for some $t_{0}=\bar{t}_{0}$

$$
M\left(\bar{t}_{0}\right)=0, \quad \frac{\mathrm{d}}{\mathrm{d} t_{0}} M\left(\bar{t}_{0}\right) \neq 0 .
$$

Then the stable and unstable manifolds of $\gamma$ intersect transversely on their energy surface, i.e., transverse homoclinic orbits to $\gamma$ exist.

The presence of such transverse homoclinic orbits implies that there exists a Smale-horseshoe and hence chaotic dynamics occurs [13,45].

Next, we consider a case in which two saddle-centers have a heteroclinic orbit in (4.1) and extend Theorem 4.1. In addition to (A1) and (A2), we assume the following.

(A2') There is another saddle-center at $(\boldsymbol{x}, \boldsymbol{y})=\left(\boldsymbol{x}_{0}^{\prime}, \mathbf{0}\right)$ with $\boldsymbol{x}_{0}^{\prime} \neq \boldsymbol{x}_{0}$ on the $\boldsymbol{x}$ plane such that the matrix $J_{1} \mathrm{D}_{x}^{2} H\left(\boldsymbol{x}_{0}^{\prime}, \mathbf{0}\right)$ has a pair of real eigenvalues $\pm \lambda^{\prime}$ and the matrix $J_{1} \mathrm{D}_{y}^{2} H\left(\boldsymbol{x}_{0}^{\prime}, \mathbf{0}\right)$ has a pair of purely imaginary eigenvalues $\pm i \omega^{\prime}\left(\lambda^{\prime}, \omega^{\prime}>0\right)$.

(A3') The two saddles $\boldsymbol{x}=\boldsymbol{x}_{0}$ and $\boldsymbol{x}_{0}^{\prime}$ in (4.2) are connected by a heteroclinic orbit $\hat{\boldsymbol{x}}^{\mathrm{h}}(t)$ such that

$$
\lim _{t \rightarrow-\infty} \hat{\boldsymbol{x}}^{\mathrm{h}}(t)=\boldsymbol{x}_{0}, \quad \lim _{t \rightarrow \infty} \hat{\boldsymbol{x}}^{\mathrm{h}}(t)=\boldsymbol{x}_{0}^{\prime} .
$$

See Fig. 1(b).

Let $\Phi^{\prime}(t)$ and $\hat{\Psi}(t)$ denote the fundamental matrices for the variational equations in the $\boldsymbol{y}$-direction around the saddle-center $\left(\boldsymbol{x}_{0}^{\prime}, \mathbf{0}\right)$ and heteroclinic orbit $\left(\hat{\boldsymbol{x}}^{\mathrm{h}}(t), \mathbf{0}\right)$ such that $\Phi^{\prime}(0)=I_{2}$. We also show that the limits

$$
\hat{B}_{-}=\lim _{t \rightarrow-\infty} \Phi(-t) \hat{\Psi}(t), \quad \hat{B}_{+}=\lim _{t \rightarrow+\infty} \Phi^{\prime}(-t) \hat{\Psi}(t)
$$

exist and set $\hat{B}_{0}=\hat{B}_{+} \hat{B}_{-}^{-1}$. Again, we define the Melnikov function $\hat{M}\left(t_{0}\right)$ as

$$
\hat{M}\left(t_{0}\right)=m\left(\boldsymbol{e}_{1}\right)-m^{\prime}\left(\hat{B}_{0} \Phi\left(t_{0}\right) \boldsymbol{e}_{1}\right),
$$

where

$$
m^{\prime}(\boldsymbol{\eta})=\frac{1}{2} \boldsymbol{\eta} \cdot \mathrm{D}_{y}^{2} H\left(\boldsymbol{x}_{0}^{\prime}, \mathbf{0}\right) \boldsymbol{\eta} .
$$

Under assumptions (A1), (A2), (A2') and (A3'), modifying arguments in the proof of Theorem 1.1 of [46] slightly, we prove the following result (see Appendix A for the proof).

Theorem 4.2. Let $\gamma$ and $\gamma^{\prime}$ be periodic orbits sufficiently close to the saddlecenters $\left(\boldsymbol{x}_{0}, \mathbf{0}\right)$ and $\left(\boldsymbol{x}_{0}^{\prime}, \mathbf{0}\right)$, respectively, on the same energy surface. Suppose that $\hat{M}\left(t_{0}\right)$ has a simple zero. Then the unstable manifold of $\gamma$ intersects the stable manifold of $\gamma^{\prime}$ transversely on the energy surface, i.e., transverse heteroclinic orbits from $\gamma$ to $\gamma^{\prime}$ exist. 
Exchanging $\boldsymbol{x}_{0}$ and $\boldsymbol{x}_{0}^{\prime}$ and applying Theorem 4.2, we can show that the unstable manifold of $\gamma^{\prime}$ intersects the stable manifold of $\gamma$ transversely on the energy surface. If the statement of the theorem holds in both the situations, then the stable and unstable manifolds of the periodic orbits form a heteroclinic cycle and consequently there exist transverse homoclinic orbits to each periodic orbit [45]. Thus, using Theorem 4.2, we can detect the occurrence of chaos when two saddle-centers have a heteroclinic connection.

As shown in $\S 3$, there exists a two-dimensional invariant plane in $\mathbb{P}_{N}\left(\sigma_{6} \pi_{\mathrm{e}}\right)$ while not in $\mathbb{P}_{N}\left(\sigma_{5} \pi_{\mathrm{e}}\right)$ and $\mathbb{P}_{N}\left(\sigma_{5} \pi_{\mathrm{o}}\right)$. Moreover, the invariant plane possesses a saddle-center equilibrium and homo- or heteroclinic orbits for a certain range of $\Gamma$, as we see for $\mathbb{P}_{6}\left(\pi_{\mathrm{e}}\right)$ in $\S 5$. Hence, some transformation recasts the Hamiltonian system (3.16) into the form (4.1) satisfying assumptions (A1)-(A3) (and (A1), (A2), (A2') and (A3')) for $\mathbb{P}_{N}\left(\sigma_{6} \pi_{\mathrm{e}}\right)$. Thus, Theorems 4.1 and 4.2 are applicable to $\mathbb{P}_{N}\left(\sigma_{6} \pi_{\mathrm{e}}\right)$ although not to $\mathbb{P}_{N}\left(\sigma_{5} \pi_{\mathrm{e}}\right)$ and $\mathbb{P}_{N}\left(\sigma_{5} \pi_{\mathrm{o}}\right)$.

\subsection{Numerical computation of criteria for chaos}

It is generally difficult to obtain analytical expressions of the homo- and heteroclinic orbits $\boldsymbol{x}^{\mathrm{h}}(t)$ and $\hat{\boldsymbol{x}}^{\mathrm{h}}(t)$ as well as those of the fundamental matrices $\Psi(t)$ and $\hat{\Psi}(t)$ of the variational equations in the $\boldsymbol{y}$-direction, all of which are required to compute the Melnikov functions (4.6) and (4.9). Hence, to estimate the Melnikov functions below, we have to rely on numerical computations including these solutions.

We consider the heteroclinic case: Assumptions (A1), (A2), (A2') and (A3') hold and two saddle-centers $(\boldsymbol{x}, \boldsymbol{y})=\left(\boldsymbol{x}_{0}, \mathbf{0}\right)$ and $\left(\boldsymbol{x}_{0}^{\prime}, \mathbf{0}\right)$ have a heteroclinic orbit $\left(\hat{\boldsymbol{x}}^{\mathrm{h}}(t), \mathbf{0}\right)$ Note that we can treat a homoclinic connection in a similar manner if we just set $\boldsymbol{x}_{0}=\boldsymbol{x}_{0}^{\prime}$. For simplicity, we assume that the matrices $\mathrm{D}_{y}^{2} H\left(\boldsymbol{x}_{0}, \mathbf{0}\right)$ and $\mathrm{D}_{y}^{2} H\left(\boldsymbol{x}_{0}^{\prime}, \mathbf{0}\right)$ are diagonal. This is the case in $\mathbb{P}_{6}\left(\pi_{\mathrm{e}}\right)$ as seen in $\S 5$. We write

$$
\mathrm{D}_{y}^{2} H\left(\boldsymbol{x}_{0}, \mathbf{0}\right)=\left(\begin{array}{cc}
m_{1} & 0 \\
0 & m_{2}
\end{array}\right) \quad \text { and } \quad \mathrm{D}_{y}^{2} H\left(\boldsymbol{x}_{0}^{\prime}, \mathbf{0}\right)=\left(\begin{array}{cc}
m_{1}^{\prime} & 0 \\
0 & m_{2}^{\prime}
\end{array}\right) .
$$

The fundamental matrix $\Phi(t)$ is given by

$$
\Phi(t)=\left(\begin{array}{cc}
\cos \omega t & \sqrt{\frac{m_{2}}{m_{1}}} \sin \omega t \\
-\sqrt{\frac{m_{1}}{m_{2}}} \sin \omega t & \cos \omega t
\end{array}\right),
$$

where $\omega=\sqrt{m_{1} m_{2}}$. The fundamental matrix $\Phi^{\prime}(t)$ is also given by (4.12) if $\omega$ is replaced with $\omega^{\prime}=\sqrt{m_{1}^{\prime} m_{2}^{\prime}}$.

Let $\boldsymbol{v}_{1}$ and $\boldsymbol{v}_{2}$ (resp. $\boldsymbol{v}_{1}^{\prime}$ and $\boldsymbol{v}_{2}^{\prime}$ ) be (column) eigenvectors for the negative and positive eigenvalues of the Jacobian matrix $J_{1} \mathrm{D}_{x}^{2} H\left(\boldsymbol{x}_{0}, \mathbf{0}\right)\left(\operatorname{resp} . J_{1} \mathrm{D}_{x}^{2} H\left(\boldsymbol{x}_{0}^{\prime}, \mathbf{0}\right)\right)$. Then the stable and unstable subspaces $E^{\mathrm{s}}$ and $E^{\mathrm{u}}$ (resp. $E^{\prime \mathrm{s}}$ and $E^{\prime \mathrm{u}}$ ) for $\boldsymbol{x}=\boldsymbol{x}_{0}$ (resp. for $\boldsymbol{x}=\boldsymbol{x}_{0}^{\prime}$ ) in (4.2) are spanned by $\boldsymbol{v}_{1}$ and $\boldsymbol{v}_{2}$ (resp. $\boldsymbol{v}_{1}^{\prime}$ and $\boldsymbol{v}_{2}^{\prime}$ ). For $\boldsymbol{x} \in \mathbb{R}^{2}$, we uniquely determine $\alpha_{j}, \alpha_{j}^{\prime} \in \mathbb{R}, j=1,2$, such that

$$
\boldsymbol{x}=\alpha_{1} \boldsymbol{v}_{1}+\alpha_{2} \boldsymbol{v}_{2}=\alpha_{1}^{\prime} \boldsymbol{v}_{1}^{\prime}+\alpha_{2}^{\prime} \boldsymbol{v}_{2}^{\prime} .
$$


Note that $\boldsymbol{x} \in E^{\mathrm{u}}$ or $E^{\mathrm{s}}$ if $\alpha_{1}=0$ or $\alpha_{2}=0$ and so on. Let $S$ and $S^{\prime}$ be $2 \times 2$ matrices whose $(j, k)$-components are given by $\boldsymbol{v}_{j} \cdot \boldsymbol{v}_{k}$ and $\boldsymbol{v}_{j}^{\prime} \cdot \boldsymbol{v}_{k}^{\prime}$, respectively, and let

$$
\left(\begin{array}{c}
\boldsymbol{l}_{\mathrm{s}}^{\mathrm{T}} \\
\boldsymbol{l}_{\mathrm{u}}^{\mathrm{T}}
\end{array}\right)=S^{-1}\left(\begin{array}{c}
\boldsymbol{v}_{1}^{\mathrm{T}} \\
\boldsymbol{v}_{2}^{\mathrm{T}}
\end{array}\right), \quad\left(\begin{array}{c}
\boldsymbol{l}_{\mathrm{s}}^{\prime \mathrm{T}} \\
\boldsymbol{l}_{\mathrm{u}}^{\prime \mathrm{T}}
\end{array}\right)=S^{\prime-1}\left(\begin{array}{c}
\boldsymbol{v}_{1}^{\prime \mathrm{T}} \\
\boldsymbol{v}_{2}^{\prime \mathrm{T}}
\end{array}\right) .
$$

Since we have $\boldsymbol{x} \cdot \boldsymbol{v}_{k}=\alpha_{1} S_{1 k}+\alpha_{2} S_{2 k}=\alpha_{1}^{\prime} S_{1 k}^{\prime}+\alpha_{2}^{\prime} S_{2 k}^{\prime}$ for $k=1,2$ due to (4.13), we have

$$
\left(\begin{array}{c}
\alpha_{1} \\
\alpha_{2}
\end{array}\right)=\left(\begin{array}{c}
\boldsymbol{l}_{\mathrm{s}}^{\mathrm{T}} \\
\boldsymbol{l}_{\mathrm{u}}^{\mathrm{T}}
\end{array}\right) \boldsymbol{x}, \quad\left(\begin{array}{c}
\alpha_{1}^{\prime} \\
\alpha_{2}^{\prime}
\end{array}\right)=\left(\begin{array}{c}
\boldsymbol{l}_{\mathrm{s}}^{\prime \mathrm{T}} \\
\boldsymbol{l}_{\mathrm{u}}^{\prime \mathrm{T}}
\end{array}\right) \boldsymbol{x} .
$$

Let $T>0$ be a sufficiently large constant. We approximate the heteroclinic orbit $\hat{\boldsymbol{x}}^{\mathrm{h}}(t)$ by solving (4.2) numerically under boundary conditions

$$
\boldsymbol{l}_{\mathrm{S}} \cdot\left(\boldsymbol{x}(-T)-\boldsymbol{x}_{0}\right)=0, \quad \boldsymbol{l}_{\mathrm{u}}^{\prime} \cdot\left(\boldsymbol{x}(T)-\boldsymbol{x}_{0}^{\prime}\right)=0,
$$

with $\boldsymbol{x}(-T)$ and $\boldsymbol{x}(T)$ sufficiently close to $\boldsymbol{x}_{0}$ and $\boldsymbol{x}_{0}^{\prime}$, since the stable and unstable manifolds are, respectively, well approximated by the stable and unstable subspaces near $\boldsymbol{x}=\boldsymbol{x}_{0}$ and $\boldsymbol{x}_{0}^{\prime}$. To obtain an approximation of the fundamental matrix $\hat{\Psi}(t)$, we numerically solve the NVE (4.4) along $\hat{\boldsymbol{x}}^{\mathrm{h}}(t)$ under two initial conditions

$$
\boldsymbol{\eta}(-T)=\boldsymbol{e}_{1} \quad \text { and } \boldsymbol{\eta}(-T)=\boldsymbol{e}_{2},
$$

where $\boldsymbol{e}_{2}=(0,1)^{\mathrm{T}}$. Finally, using the approximations

$$
\hat{B}_{-}=\Phi(T), \quad \hat{B}_{+}=\Phi^{\prime}(-T) \hat{\Psi}(T), \quad \hat{B}_{0}=\Phi^{\prime}(-T) \hat{\Psi}(T) \Phi(-T),
$$

we compute (4.9) as follows:

$$
\begin{aligned}
\hat{M}\left(t_{0}\right)= & \frac{1}{2} m_{1}-\frac{1}{2} m_{1}^{\prime}\left(\hat{b}_{11} \cos \omega t_{0}-\hat{b}_{12} \sqrt{\frac{m_{1}}{m_{2}}} \sin \omega t_{0}\right)^{2} \\
& -\frac{1}{2} m_{2}^{\prime}\left(\hat{b}_{21} \cos \omega t_{0}-\hat{b}_{22} \sqrt{\frac{m_{1}}{m_{2}}} \sin \omega t_{0}\right)^{2} \\
= & \frac{1}{2}\left[\left(m_{1}-\beta_{0}\right)-\beta_{1} \cos \left(2 \omega t_{0}+\tau\right)\right],
\end{aligned}
$$

where $\tau \in[0,2 \pi)$ is some constant, $\hat{b}_{j k}$ is the $(j, k)$-component of the matrix $\hat{B}_{0}$ and

$$
\begin{aligned}
& \beta_{0}=\frac{1}{2 m_{2}}\left(\hat{b}_{12}^{2} m_{1} m_{1}^{\prime}+\hat{b}_{11}^{2} m_{2} m_{1}^{\prime}+\hat{b}_{22}^{2} m_{1} m_{2}^{\prime}+\hat{b}_{21}^{2} m_{2} m_{2}^{\prime}\right), \\
& \beta_{1}=\sqrt{\beta_{0}^{2}-\frac{m_{1} m_{1}^{\prime} m_{2}^{\prime}}{m_{2}}}
\end{aligned}
$$

Here we used the fact that $\left|\hat{B}_{0}\right|=\hat{b}_{11} \hat{b}_{22}-\hat{b}_{12} \hat{b}_{21}=1$ via the Liouville theorem (e.g., $\S 27$ of [2]) for (4.3) and (4.4) since $\operatorname{tr}\left(J_{1} \mathrm{D}_{y}^{2} H\left(\hat{\boldsymbol{x}}^{\mathrm{h}}(t), \mathbf{0}\right)\right)=0$. Hence, if $\beta_{1}>\left|m_{1}-\beta_{0}\right|$, i.e.,

$$
\begin{aligned}
\hat{\Delta} & =\frac{m_{2}}{m_{1}}\left[\beta_{1}^{2}-\left(m_{1}-\beta_{0}\right)^{2}\right] \\
& =\hat{b}_{12}^{2} m_{1} m_{1}^{\prime}+\hat{b}_{11}^{2} m_{2} m_{1}^{\prime}+\hat{b}_{22}^{2} m_{1} m_{2}^{\prime}+\hat{b}_{21}^{2} m_{2} m_{2}^{\prime}-m_{1} m_{2}-m_{1}^{\prime} m_{2}^{\prime}>0
\end{aligned}
$$


then the Melnikov function $\hat{M}\left(t_{0}\right)$ has a simple zero and by Theorem 4.2 the unstable manifolds of periodic orbits near $\left(\boldsymbol{x}_{0}, \mathbf{0}\right)$ intersect the stable manifolds of periodic orbits near $\left(\boldsymbol{x}_{0}^{\prime}, \mathbf{0}\right)$ transversely.

To compute (4.20), we numerically solve (4.2) and (4.4) under the boundary conditions (4.16) and (4.17), respectively, and calculate the matrix $\hat{B}$ by (4.18). Numerical solutions of the boundary value problem along with estimates of $\hat{B}$ and $\hat{\Delta}$ are easily continued with the parameter $\Gamma$ by the numerical continuation tool "AUTO97" [7], if once a solution is obtained for a special value of $\Gamma$. To obtain the starting solution, we take a point that is very close to $\boldsymbol{x}_{0}$ and satisfies the first equation of (4.16) as $\boldsymbol{x}(-T)$, and solve (4.2) and (4.17) as an initial value problem using an adequate numerical method. See $\S 5$ for more details on our concrete approach.

For the case of the homoclinic orbit $\boldsymbol{x}^{\mathrm{h}}(t)$, setting $\boldsymbol{x}_{0}^{\prime}=\boldsymbol{x}_{0}$ and applying the same argument, we see that if

$$
\Delta=b_{12}^{2} m_{1}^{2}+\left(b_{11}^{2}+b_{22}^{2}\right) m_{1} m_{2}+b_{21}^{2} m_{2}^{2}-2 m_{1} m_{2}>0,
$$

where $b_{j k}$ is the $(j, k)$-component of the matrix $B_{0}$, then $M\left(t_{0}\right)$ has a simple zero and by Theorem 4.1 the stable and unstable manifolds of periodic orbits near $\left(\boldsymbol{x}_{0}, \mathbf{0}\right)$ intersect transversely. We also use "AUTO97" to compute the criterion (4.21) in $\S 5$. In Appendix B the accuracy of our numerical approach is demonstrated for a generalized version of the Hénon-Heiles system [16], in which the Melnikov analysis can be performed analytically.

\subsection{Numerical computation of stable and unstable manifolds}

As stated in $\S 4.1$, the Melnikov approach cannot be applied to $\mathbb{P}_{N}\left(\sigma_{5} \pi_{\mathrm{e}}\right)$ and $\mathbb{P}_{N}\left(\sigma_{5} \pi_{\mathrm{e}}\right)$ since they contain no invariant plane. However, even in those cases, we can compute the stable and unstable manifolds of periodic orbits near saddlecenters by the numerical method of [50]. Using this approach, we show that the stable and unstable manifolds intersect transversely and chaotic dynamics occurs for $\mathbb{P}_{6}\left(\pi_{\mathrm{e}}\right)$ and $\mathbb{P}_{5}\left(\pi_{\mathrm{o}}\right) \mathrm{n} \S \S 5$ and 6 . We briefly review the numerical method in our context.

Rewrite the Hamiltonian systems for $\mathbb{P}_{5}\left(\pi_{\mathrm{o}}\right)$ and $\mathbb{P}_{6}\left(\pi_{\mathrm{e}}\right)$ as

$$
\dot{\boldsymbol{z}}=J_{2} \mathrm{D} H(\boldsymbol{z}),
$$

where $z=\left(Q_{3}, Q_{4}, P_{3}, P_{4}\right)$ or $\left(x_{1}, y_{1}, x_{2}, y_{2}\right), H: \mathbb{R}^{4} \rightarrow \mathbb{R}$ is the Hamiltonian function and $J_{2}$ is the $4 \times 4$ symplectic matrix,

$$
J_{2}=\left(\begin{array}{cc}
0 & I_{2} \\
-I_{2} & 0
\end{array}\right)
$$

Recall that $I_{2}$ denotes the $2 \times 2$ identity matrix.

As in [6], to compute a periodic orbit $\boldsymbol{z}=\overline{\boldsymbol{z}}(t)$ near the saddle-center $\boldsymbol{z}=\boldsymbol{z}_{\mathrm{sc}}$, we solve (4.22) under the periodic boundary condition

$$
\overline{\boldsymbol{z}}(0)=\overline{\boldsymbol{z}}(\bar{T}),
$$


with $\bar{T}$ the period of the periodic orbit, while its phase is fixed at

$$
\bar{z}_{4}(0)=z_{40},
$$

with $z_{j}$ the $j$-th component of $z$, and the Hamiltonian constrained to be constant

$$
H(\bar{z}(0))=h,
$$

where $\bar{T}$ is the period of the periodic orbit. We compute a trajectory $\boldsymbol{z}=\boldsymbol{z}^{\mathrm{u}}(t)$ on the unstable manifold of the periodic orbit $\overline{\boldsymbol{z}}(t)$ by solving (4.22) under the boundary conditions

$$
\boldsymbol{z}^{\mathrm{u}}(0)-\overline{\boldsymbol{z}}(0)=\varepsilon_{\mathrm{u}} \boldsymbol{e}^{\mathrm{u}}, \quad \boldsymbol{z}^{\mathrm{u}}\left(T_{\mathrm{u}}\right)=\boldsymbol{z}_{0}^{\mathrm{u}},
$$

where $\boldsymbol{e}^{\mathrm{u}} \in \mathbb{R}^{4}$ is an unstable eigenvector with unit modulus of the monodromy matrix for $\overline{\boldsymbol{z}}(t), \varepsilon_{\mathrm{u}} \ll 1$ and $T_{\mathrm{u}}$ are positive constants, and $\boldsymbol{z}_{0}^{\mathrm{u}} \in \mathbb{R}^{4}$ is a constant vector and represents an approximate point on the unstable manifold. Thus, numerical continuation of the solutions $\overline{\boldsymbol{z}}(t)$ and $\boldsymbol{z}^{\mathrm{u}}(t)$ for the boundary value problem (4.22)-(4.26) gives the unstable manifold. Similarly, we compute the stable manifold by continuing the solution $\overline{\boldsymbol{z}}(t)$ of (4.22) satisfying (4.23)-(4.25) and a solution $\boldsymbol{z}^{\mathrm{s}}(t)$ of (4.22) satisfying the boundary conditions

$$
\boldsymbol{z}^{\mathrm{S}}(0)-\overline{\boldsymbol{z}}(0)=\varepsilon_{\mathrm{s}} \boldsymbol{e}^{\mathrm{s}}, \quad \boldsymbol{z}^{\mathrm{S}}\left(-T_{\mathrm{S}}\right)=\boldsymbol{z}_{0}^{\mathrm{s}},
$$

where $\boldsymbol{e}^{\mathrm{s}} \in \mathbb{R}^{4}$ is a stable eigenvector with unit modulus of the monodromy matrix for $\overline{\boldsymbol{z}}(t), \varepsilon_{\mathrm{S}} \ll 1$ and $T_{\mathrm{S}}$ are positive constants, and a constant vector $\boldsymbol{z}_{0}^{\mathrm{s}} \in$ $\mathbb{R}^{4}$ represents an approximate point on the stable manifold. In the continuations $\bar{T}, T_{\mathrm{u}, \mathrm{s}}, \varepsilon_{\mathrm{u}, \mathrm{s}}, z_{40}$ or $\boldsymbol{z}_{0}^{\mathrm{u}, \mathrm{s}}$ can be taken as the free parameters.

In the numerical computations of $\S \S 5$ and 6 , we make a small modification in the standard version of "AUTO97" so that the monodromy matrices of periodic orbits are provided at each step in the computation, as in [6]. Without the modification, we have to solve the $4 \times 4$ matrix version of the four-dimensional, linearized system of (4.25) around the periodic orbits to obtain the monodromy matrices. Owing to this modification, the dimension of the system to be solved is decreased by 16 , so that we just solve an eight-dimensional system to compute the periodic orbits and orbits on its stable or unstable manifolds. As the starting solutions for the continuations by "AUTO97", we take solutions of the linearized system

$$
\dot{\boldsymbol{\zeta}}=J_{2} \mathrm{D}^{2} H\left(\boldsymbol{z}_{\mathrm{sc}}\right) \boldsymbol{\zeta}
$$

with the following forms:

$$
\overline{\boldsymbol{z}}(t)=\boldsymbol{z}_{\mathrm{sc}}+\varepsilon_{c}\left(\boldsymbol{e}_{0}^{+c} e^{i \omega t}+\boldsymbol{e}_{0}^{-c} e^{-i \omega t}\right), \quad t \in[0, \bar{T}],
$$

and

$$
\boldsymbol{z}^{\mathrm{u}}(t)=\overline{\boldsymbol{z}}(t)+\varepsilon_{\mathrm{u}} \boldsymbol{e}_{0}^{\mathrm{u}} e^{\lambda_{\mathrm{u}} t}, \quad t \in\left[0, T_{\mathrm{u}}\right]
$$

or

$$
\boldsymbol{z}^{\mathrm{s}}(t)=\overline{\boldsymbol{z}}(t)+\varepsilon_{\mathrm{s}} \boldsymbol{e}_{0}^{\mathrm{s}} e^{\lambda_{\mathrm{s}} t}, \quad t \in\left[-T_{\mathrm{s}}, 0\right],
$$


where $\lambda_{\mathrm{s}}, \lambda_{\mathrm{u}}$ and $\pm i \omega$ are the stable, unstable and center eigenvalues of $J_{2} \mathrm{D}^{2} H\left(\boldsymbol{z}_{\mathrm{sc}}\right)$, $\boldsymbol{e}_{0}^{\mathrm{s}}, \boldsymbol{e}_{0}^{\mathrm{u}}$ and $\boldsymbol{e}_{0}^{ \pm c}$ are the associated eigenvectors for $\lambda_{\mathrm{s}}, \lambda_{\mathrm{u}}$ and $\pm i \omega$ with unit moduli such that $\operatorname{Re}\left(\boldsymbol{e}_{0}^{+c}\right)=\operatorname{Re}\left(\boldsymbol{e}_{0}^{-c}\right)$, and $\varepsilon_{c}>0$ and $T_{\mathrm{u}, \mathrm{s}}$ are small constants. Equations (4.30) and (4.31) represent solutions of (4.28) such that $\boldsymbol{z}^{\mathrm{u}}(t) \rightarrow \overline{\boldsymbol{z}}(t)$ as $t \rightarrow-\infty$ and $\boldsymbol{z}^{\mathrm{s}}(t) \rightarrow \overline{\boldsymbol{z}}(t)$ as $t \rightarrow \infty$, as well as Eq. (4.29) represents a small periodic solution of (4.28) near $\boldsymbol{z}_{\mathrm{sc}}$. Note that $\bar{T}=2 \pi / \omega$ and $\lambda_{\mathrm{s}}=-\lambda_{\mathrm{u}}$ since the system (4.22) is Hamiltonian. The values of $\varepsilon_{c}=10^{-6}, \varepsilon_{\mathrm{u}, \mathrm{s}}=10^{-4}$ and $T_{\mathrm{u}, \mathrm{s}}=10^{-2}$ are typically chosen in our numerical computations.

\section{Detection of chaos in $\mathbb{P}_{6}\left(\pi_{\mathrm{e}}\right)$}

We apply the analytical and numerical techniques of $\S 4$ to $\mathbb{P}_{6}\left(\pi_{\mathrm{e}}\right)$. As stated at the ends of $\S 2, \mathbb{P}_{6}\left(\pi_{\mathrm{e}}\right)$ has the invariant plane $\mathbb{P}_{6}\left(\sigma_{3} \pi_{\mathrm{e}}\right)$, on which there exist saddle-centers connected by homo- and/or heteroclinic orbits, as seen below.

We first introduce the following new symplectic transformation so that assumption (A1) holds and the $\boldsymbol{x}$-plane is invariant under the flow of (4.1):

$$
x_{1}=Q_{3}, \quad x_{2}=P_{3}-\frac{1}{2} P_{4}, \quad y_{1}=Q_{3}+2 Q_{4}-\pi, \quad y_{2}=\frac{1}{2} P_{4} .
$$

Under the above transformation (5.1), the Hamiltonian (3.16) with $n=1$ becomes

$$
\begin{aligned}
H(\boldsymbol{x}, \boldsymbol{y})= & -\frac{1}{2 \pi} \log \left[1-\sqrt{1-\left(x_{2}+y_{2}\right)^{2}} \sin \frac{1}{2}\left(x_{1}+y_{1}\right)\right] \\
& -\frac{1}{2 \pi} \log \left[1+\sqrt{1-\left(x_{2}+y_{2}\right)^{2}} \sin \frac{1}{2}\left(x_{1}+y_{1}\right)\right] \\
& -\frac{1}{2 \pi} \log \left[1-\sqrt{1-\left(x_{2}-y_{2}\right)^{2}} \sin \frac{1}{2}\left(x_{1}-y_{1}\right)\right] \\
& -\frac{1}{2 \pi} \log \left[1+\sqrt{1-\left(x_{2}-y_{2}\right)^{2}} \sin \frac{1}{2}\left(x_{1}-y_{1}\right)\right] \\
& -\frac{1}{2 \pi} \log \left[1+\left(x_{2}^{2}-y_{2}^{2}\right)-\sqrt{\left(1-\left(x_{2}+y_{2}\right)^{2}\right)\left(1-\left(x_{2}-y_{2}\right)^{2}\right)} \cos x_{1}\right] \\
& -\frac{1}{2 \pi} \log \left[1-\left(x_{2}^{2}-y_{2}^{2}\right)+\sqrt{\left(1-\left(x_{2}+y_{2}\right)^{2}\right)\left(1-\left(x_{2}-y_{2}\right)^{2}\right)} \cos y_{1}\right] \\
& -\frac{1}{4 \pi} \log \left[1+\left(x_{2}+y_{2}\right)^{2}+\left(1-\left(x_{2}+y_{2}\right)^{2}\right) \cos \left(x_{1}+y_{1}\right)\right] \\
& -\frac{1}{4 \pi} \log \left[1+\left(x_{2}-y_{2}\right)^{2}+\left(1-\left(x_{2}-y_{2}\right)^{2}\right) \cos \left(x_{1}-y_{1}\right)\right] \\
& -\frac{\Gamma}{2 \pi} \log \left[\left(1-\left(x_{2}-y_{2}\right)^{2}\right)\left(1-\left(x_{2}+y_{2}\right)^{2}\right)\right] .
\end{aligned}
$$


(a) $\Gamma=2.0$

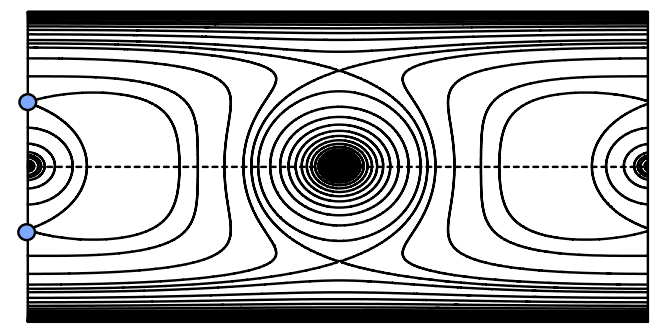

(b) $\Gamma=1.25$

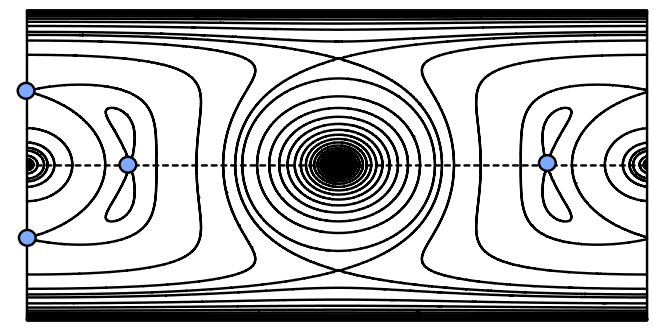

(c) $\Gamma=1.00$

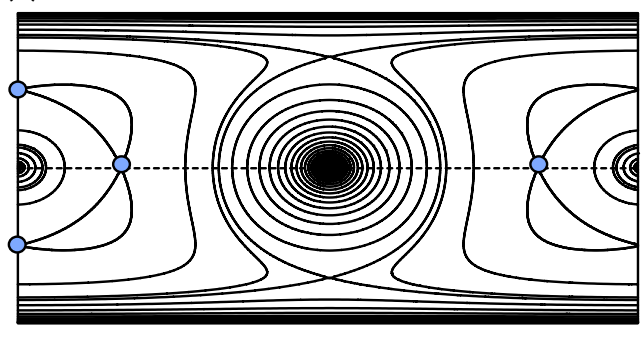

(d) $\Gamma=0.75$

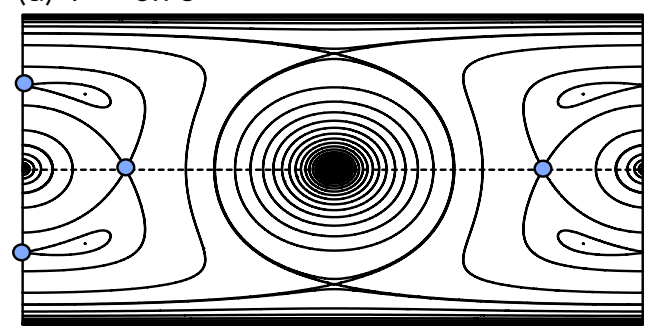

(e) $\Gamma=0.25$

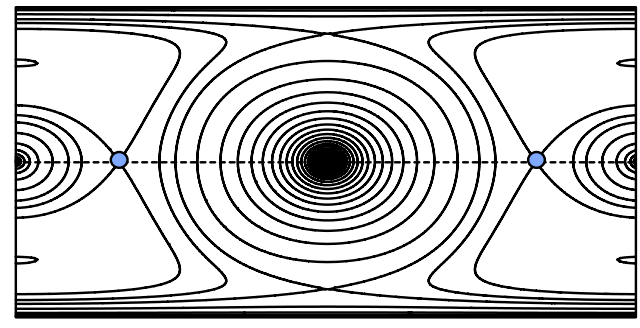

(f) $\Gamma=-0.01$

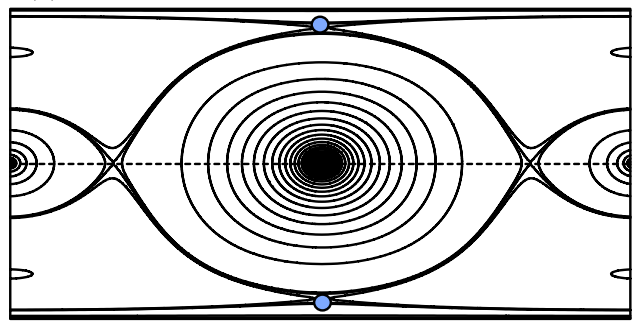

Figure 2: Phase portraits of the system restricted on the $\boldsymbol{x}$-plane (4.2) for $\mathbb{P}_{6}\left(\pi_{\mathrm{e}}\right)$. The positions of saddle-centers are plotted as the filled circles $\bullet$.

On the $\boldsymbol{x}$-plane the Hamiltonian (5.2) becomes

$$
\begin{aligned}
& H(\boldsymbol{x}, \mathbf{0}) \\
&=-\frac{1}{\pi} \log \left(1-\sqrt{1-x_{2}^{2}} \sin \frac{x_{1}}{2}\right)-\frac{1}{\pi} \log \left(1+\sqrt{1-x_{2}^{2}} \sin \frac{x_{1}}{2}\right) \\
&-\frac{1}{2 \pi} \log \left(1+x_{2}^{2}-\left(1-x_{2}^{2}\right) \cos x_{1}\right)-\frac{1}{2 \pi} \log \left(1+x_{2}^{2}+\left(1-x_{2}^{2}\right) \cos x_{1}\right) \\
&-\frac{1}{2 \pi} \log 2\left(1-x_{2}^{2}\right)-\frac{\Gamma}{\pi} \log \left(1-x_{2}^{2}\right),
\end{aligned}
$$

and the $\boldsymbol{x}$-component system (4.2) has hyperbolic saddles

(i) at $\boldsymbol{s}_{1}=\left(\frac{1}{3} \pi, 0\right)$ and $\boldsymbol{s}_{2}=\left(\frac{5}{3} \pi, 0\right)$ for $\Gamma<\frac{3}{2}$;

(ii) at $s_{3}=\left(\pi, \sqrt{\frac{3}{2(\Gamma+2)}}\right)$ and $s_{4}=\left(\pi,-\sqrt{\frac{3}{2(\Gamma+2)}}\right)$ for $\Gamma>-\frac{1}{2}$; 
Table 1: Homoclinic or heteroclinic connections between saddle-centers on the $\boldsymbol{x}$-plane in $\mathbb{P}_{6}\left(\pi_{\mathrm{e}}\right)$. The symbol $\boldsymbol{s}_{p} \leftrightarrow \boldsymbol{s}_{q}$ indicates that $\boldsymbol{s}_{p}$ and $\boldsymbol{s}_{q}$ have a heteroclinic $(p \neq q)$ or a homoclinic $(p=q)$ connection.

\begin{tabular}{c|c|c|c}
\hline \hline & $\Gamma$ & homo- or heteroclinic connections & Fig. 2 \\
\hline ia' & $1<\Gamma<\frac{3}{2}$ & $\boldsymbol{s}_{1} \leftrightarrow \boldsymbol{s}_{1}, \boldsymbol{s}_{2} \leftrightarrow \boldsymbol{s}_{2}$ & (b) \\
ib' & $0<\Gamma<1$ & $\boldsymbol{s}_{1} \leftrightarrow \boldsymbol{s}_{2}$ & (d), (e) \\
ii' & $-\frac{1}{4}(5-\sqrt{15})<\Gamma<0$ & $\boldsymbol{s}_{3} \leftrightarrow \boldsymbol{s}_{3}, \boldsymbol{s}_{4} \leftrightarrow \boldsymbol{s}_{4}$ & (f) \\
iiia' & $\Gamma>1$ & $\boldsymbol{s}_{5} \leftrightarrow \boldsymbol{s}_{6}$ & (a), (b) \\
iiib' & $\frac{1}{2}<\Gamma<1$ & $\boldsymbol{s}_{5} \leftrightarrow \boldsymbol{s}_{5}, \boldsymbol{s}_{6} \leftrightarrow \boldsymbol{s}_{6}$ & (d) \\
iv' & $\Gamma=1$ & $\boldsymbol{s}_{1} \leftrightarrow \boldsymbol{s}_{5}, \boldsymbol{s}_{1} \leftrightarrow \boldsymbol{s}_{6} \boldsymbol{s}_{2} \leftrightarrow \boldsymbol{s}_{5}, \boldsymbol{s}_{2} \leftrightarrow \boldsymbol{s}_{6}$ & (c) \\
\hline \hline
\end{tabular}

(iii) at $s_{5}=\left(0, \sqrt{\frac{1}{2(\Gamma+1)}}\right)$ and $s_{6}=\left(0,-\sqrt{\frac{1}{2(\Gamma+1)}}\right)$ for $\Gamma>\frac{1}{2}$.

These hyperbolic saddles correspond to equilibria in (4.1) and have homo- or heteroclinic orbits. The phase portraits of (4.2) are displayed in Fig. 2 for several values of $\Gamma$. They also indicate the dynamics on the invariant plane $\mathbb{P}_{6}\left(\sigma_{3} \pi_{\mathrm{e}}\right)$. As required in assumptions (A2) and (A2'), these equilibria are saddle-centers in $(4.1)$

(i') when $0<\Gamma<\frac{3}{2}$ for $s_{1}$ and $s_{2}$;

(ii') when $-\frac{1}{4}(5-\sqrt{15})(=-0.28175 \ldots)<\Gamma<0$ for $s_{3}$ and $s_{4}$;

(iii') when $\Gamma>\frac{1}{2}$ for $\boldsymbol{s}_{5}$ and $\boldsymbol{s}_{6}$.

Finally, we see that assumption (A3) or (A3') holds as follows:

(ia') For $1<\Gamma<\frac{3}{2}$ there are a pair of homoclinic orbits to each of $\boldsymbol{s}_{1}$ and $\boldsymbol{s}_{2}$;

(ib') for $0<\Gamma<1$ there are two heteroclinic cycles between $s_{1}$ and $\boldsymbol{s}_{2}$;

(ii') for $-\frac{1}{4}(5-\sqrt{15})<\Gamma<0$ there are a pair of homoclinic orbits to each of $s_{3}$ and $s_{4}$

(iiia') for $\Gamma>1$ there are two heteroclinic cycles between $\boldsymbol{s}_{5}$ and $\boldsymbol{s}_{6}$;

(iiib') for $\frac{1}{2}<\Gamma<1$ there are a pair of homoclinic orbits to each of $\boldsymbol{s}_{5}$ and $\boldsymbol{s}_{6}$;

(iv') for $\Gamma=1$ there are four heteroclinic cycles between $\boldsymbol{s}_{1}$ or $\boldsymbol{s}_{2}$ and $\boldsymbol{s}_{5}$ or $\boldsymbol{s}_{6}$.

We give a summary of this statement in Table 1 .

For each of these cases we apply the numerical method of $\S 4.2$ to compute the conditions (4.20) and (4.21). See Appendix B for the accuracy of our numerical 
method. First, we have each component of the second derivative $\mathrm{D}_{y}^{2} H(\boldsymbol{x}, \mathbf{0})$ as

$$
\begin{aligned}
& \frac{\partial^{2} H}{\partial y_{1}^{2}}(\boldsymbol{x}, \mathbf{0})=\frac{15 x_{2}^{4}-22 x_{2}^{2}+15+16\left(1-x_{2}^{4}\right) \cos x_{1}+\left(1-x_{2}^{2}\right)^{2} \cos 2 x_{1}}{8 \pi\left(1+x_{2}^{2}+\left(1-x_{2}^{2}\right) \cos x_{1}\right)^{2}}, \\
& \frac{\partial^{2} H}{\partial y_{1} \partial y_{2}}(\boldsymbol{x}, \mathbf{0})=-\frac{6 x_{2} \sin x_{1}}{\pi\left(1+x_{2}^{2}+\left(1-x_{2}^{2}\right) \cos x_{1}\right)^{2}}, \\
& \frac{\partial^{2} H}{\partial y_{2}^{2}}(\boldsymbol{x}, \mathbf{0})=\frac{\mu_{0}\left(x_{2}\right)+\mu_{1}\left(x_{2}\right) \cos x_{1}+\mu_{2}\left(x_{2}\right) \cos 2 x_{1}+\mu_{3}\left(x_{2}\right) \cos 3 x_{1}}{4 \pi\left(1-x_{2}^{2}\right)^{2}\left(1+x_{2}^{2}-\left(1-x_{2}^{2}\right) \cos x_{1}\right)\left(1+x_{2}^{2}+\left(1-x_{2}^{2}\right) \cos x_{1}\right)^{2}},
\end{aligned}
$$

where

$\mu_{0}\left(x_{2}\right)=2\left[(2 \Gamma+5) x_{2}^{8}+(16 \Gamma+9) x_{2}^{6}+(28 \Gamma-11) x_{2}^{4}+(16 \Gamma+15) x_{2}^{2}+2(\Gamma-1)\right]$,

$\mu_{1}\left(x_{2}\right)=\left(1-x 2^{2}\right)\left[(2 \Gamma+5) x_{2}^{6}+6(5 \Gamma+7) x_{2}^{4}+(30 \Gamma-67) x_{2}^{2}+2(\Gamma+2)\right]$,

$\mu_{2}\left(x_{2}\right)=-2\left(1-x_{2}^{2}\right)^{2}\left[(2 \Gamma+5) x_{2}^{4}+(4 \Gamma-5) x_{2}^{2}+2(\Gamma-1)\right]$,

$\mu_{3}\left(x_{2}\right)=-\left(1-x_{2}^{2}\right)^{3}\left[(2 \Gamma+5) x_{2}^{2}+2(\Gamma+2)\right]$.

At $\boldsymbol{x}=\boldsymbol{s}_{j}$ for $j=1,2, \ldots, 6$, the matrix $\mathrm{D}_{y}^{2} H(\boldsymbol{x}, \mathbf{0})$ is diagonal and has the form of (4.11) with

$$
m_{1}=\frac{5}{4 \pi}, \quad m_{2}=\frac{2 \Gamma}{\pi},
$$

for $\boldsymbol{x}=s_{1}, s_{2}$

$$
m_{1}=-\frac{\Gamma}{2 \pi}, \quad m_{2}=\frac{2(\Gamma+2)\left(8 \Gamma^{2}+20 \Gamma+5\right)}{\pi(2 \Gamma+1)^{2}},
$$

for $\boldsymbol{x}=\boldsymbol{s}_{3}, \boldsymbol{s}_{4} ;$ and

$$
m_{1}=\frac{8 \Gamma+5}{8 \pi(\Gamma+1)}, \quad m_{2}=\frac{8 \Gamma(\Gamma+1)^{2}}{\pi(2 \Gamma+1)^{2}},
$$

for $\boldsymbol{x}=s_{5}, s_{6}$. To obtain a starting solution for the boundary value problem (4.2), (4.4), (4.16) and (4.17), we use a Fortran code called "DOP853" [14] to solve (4.2) and (4.4) as an initial value problem as stated at the end of $\S 4.2$. The code is based on the explicit Runge-Kutta method of order 8 by Dormand and Prince [8], and a fifth order error estimator with third order correction is utilized. It also has a dense output of order 7. See [14] for more details on the code. A small tolerance of $10^{-8}$ or $10^{-9}$ is chosen in the computations so that the numerical results are very accurate. For instance, the value of the Hamiltonian energy changes by only $10^{-15}$ at most for the numerical solutions.

Figure 3 shows the computational results of $\Delta$ and $\hat{\Delta}$. We see that $\Delta$ and $\hat{\Delta}$ are positive except for $\Gamma=1$. This indicates by Theorems 4.1 and 4.2 that there exist transverse homoclinic orbits to periodic orbits near the saddle-centers and chaotic dynamics occurs for $\Gamma \neq 1$. On the other hand, $\hat{\Delta}$ is negative for a heteroclinic orbit connecting $s_{5}$ to $s_{1}$ at $\Gamma=1$, which means that the unstable manifold of periodic orbits near the saddle-center $\left(\boldsymbol{s}_{1}, \mathbf{0}\right)$ does not intersect the stable manifold of periodic orbits near $\left(s_{5}, \mathbf{0}\right)$. Thus, the Melnikov theory says nothing about the occurrence of chaos for $\Gamma=1$. 

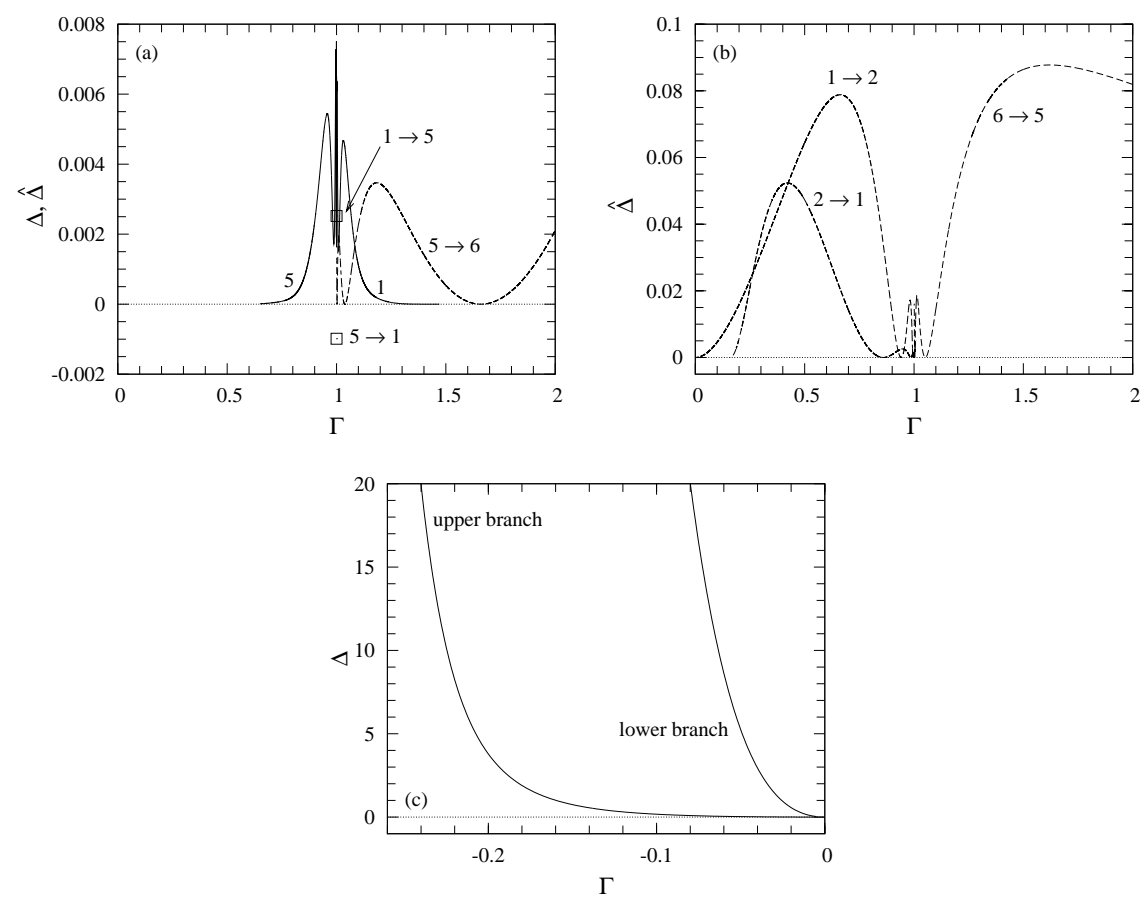

Figure 3: Plots of $\Delta$ and $\hat{\Delta}$ computed for the saddle-centers in $\mathbb{P}_{6}\left(\pi_{\mathrm{e}}\right)$ : (a) Homo- and heteroclinic orbits to $s_{1,5,6}$; (b) heteroclinic orbits to $s_{1,2,5,6}$; (c) upper and lower homoclinic orbits to $s_{3} . \Delta$ is plotted as solid lines, while $\hat{\Delta}$ is plotted as dotted lines or " $\square$ ". The labels represent the subscripts of equilibria which the associated homo- or heteroclinic orbits connect. Note that $\Delta$ has the same value for homoclinic orbits to $s_{2 k}$ and $s_{2 k-1}$ and $\hat{\Delta}$ has the same positive (resp. negative) value for heteroclinic orbits from $s_{1}$ or $s_{2}$ to $s_{5}$ or $\boldsymbol{s}_{6}$ (resp. from $s_{5}$ or $s_{6}$ to $s_{1}$ or $s_{2}$ ).

To support the above results, we compute the stable and unstable manifolds of periodic orbits near saddle-centers with the numerical method of $\S 4.3$. Figures 4 and 5 , respectively, show periodic orbits near the saddle-center $\left(\boldsymbol{s}_{1}, \mathbf{0}\right)$ for $\Gamma=1.25$, and the stable and unstable manifolds of periodic orbits on the Poincaré section, $\left\{(\boldsymbol{x}, \boldsymbol{y}) \in \mathbb{R}^{2} \times \mathbb{R}^{2} \mid y_{2}=0\right\}$, for $\Gamma=1.25$ and 1. In Fig. 5(a), the stable and unstable manifolds of the outermost periodic orbit in Fig. 4 intersect transversely. In Fig. 5(b), the stable and unstable manifolds of periodic orbits near $\left(\boldsymbol{s}_{1}, \mathbf{0}\right)$ and $\left(\boldsymbol{s}_{5}, \mathbf{0}\right)$ are displayed when the saddle-centers have heteroclinic connections at $\Gamma=1$ (see Fig. 2(c)). We see that the unstable (resp. stable) manifold of the periodic orbit near $\left(s_{1}, \mathbf{0}\right)$ intersects transversely (resp. does not intersect) the stable (resp. unstable) manifold of periodic orbit near $\left(s_{5}, \mathbf{0}\right)$, as our theory predicts. However, since the stable and unstable manifolds of the periodic orbit near $\left(\boldsymbol{s}_{1}, \mathbf{0}\right)$ intersect transversely as observed in Fig. 5(b), chaotic dynamics also occurs for $\Gamma=1$.

Finally, we give some direct numerical simulations for chaotic motions. Figure 6 shows projections onto the $\boldsymbol{x}$-plane of trajectories on the Poincaré section 

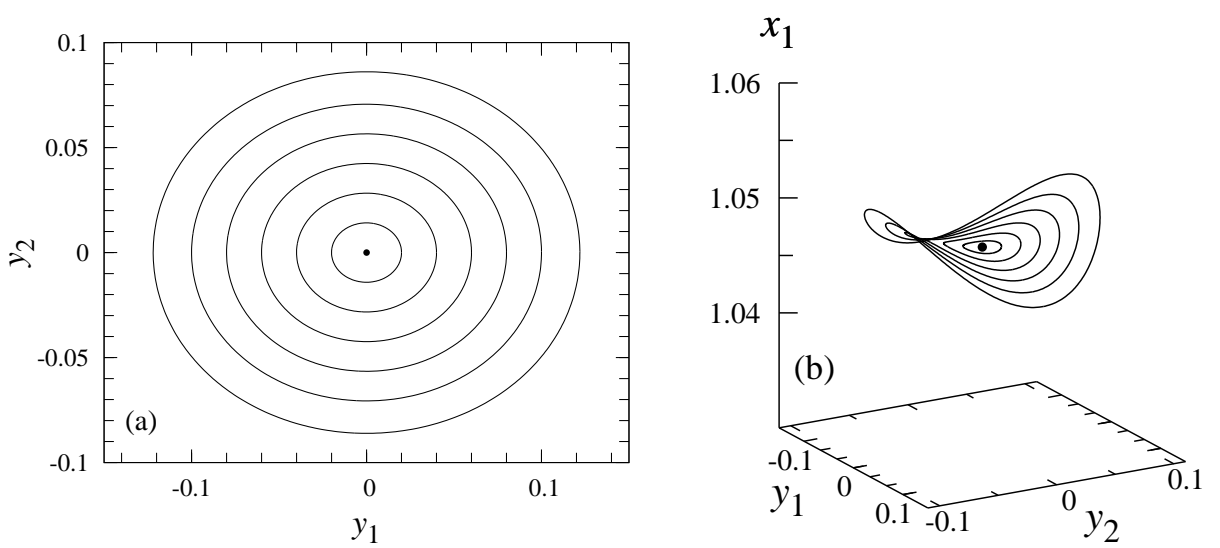

Figure 4: Periodic orbits near the saddle-center $\left(s_{1}, 0\right)$ for $\Gamma=1.25$ in $\mathbb{P}_{6}\left(\pi_{\mathrm{e}}\right)$ : (a) Their projections onto the $\boldsymbol{y}$-plane; (b) onto the $\left(y_{1}, y_{2}, x_{1}\right)$-space. The outermost periodic orbit has Hamiltonian energy of $H=0.03$.
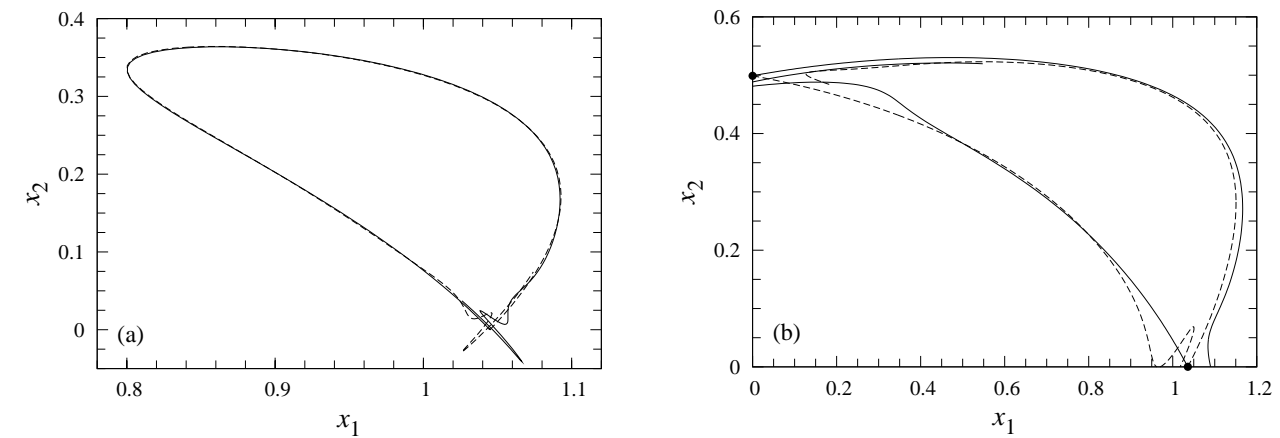

Figure 5: Stable and unstable manifolds of periodic orbits on the Poincaré section $\left\{y_{2}=0\right\}$ for $\mathbb{P}_{6}\left(\pi_{\mathrm{e}}\right)$ : (a) For a periodic orbit near $\left(\boldsymbol{s}_{1}, \mathbf{0}\right)$ for $\Gamma=1.25$ and $H=0.03$; (b) for periodic orbits near $\left(\boldsymbol{s}_{1}, \mathbf{0}\right)$ and $\left(\boldsymbol{s}_{5}, \mathbf{0}\right)$ for $\Gamma=1$ and $H=$ 0.04. The solid and broken lines represent the stable and unstable manifolds, respectively. In plate (b) the point "•" represents the periodic orbits on the Poincaré section.

$\left\{y_{2}=0\right\}$ with initial data near the saddle-centers. To obtain the cross-section points from the trajectories, an interval $\left[t_{n-1}, t_{n}\right]$ of numerical integration such that $y_{2}\left(t_{n-1}\right)<0$ and $y_{2}\left(t_{n}\right) \geq 0$ is searched and the secant method is applied to the dense output for the interval with a tolerance of $\left|y_{2}\right|<10^{-8}$. All these computations are performed by a software called "Dynamics" [32] into which the C version of the code "DOP853" [14] is built in a manner explained in [49]. We also show actual chaotic trajectories starting near the saddle-centers on the sphere in Fig. 7, which correspond to the Poincaré sections in Fig. 6. Both of them clearly illustrate chaotic behavior near the homo- or heteroclinic orbits to the saddle-centers. Further information on the trajectories in Figs. 6 and 7 are given in Table 2. Note that the chaotic trajectory for $\Gamma=-0.1$ in 

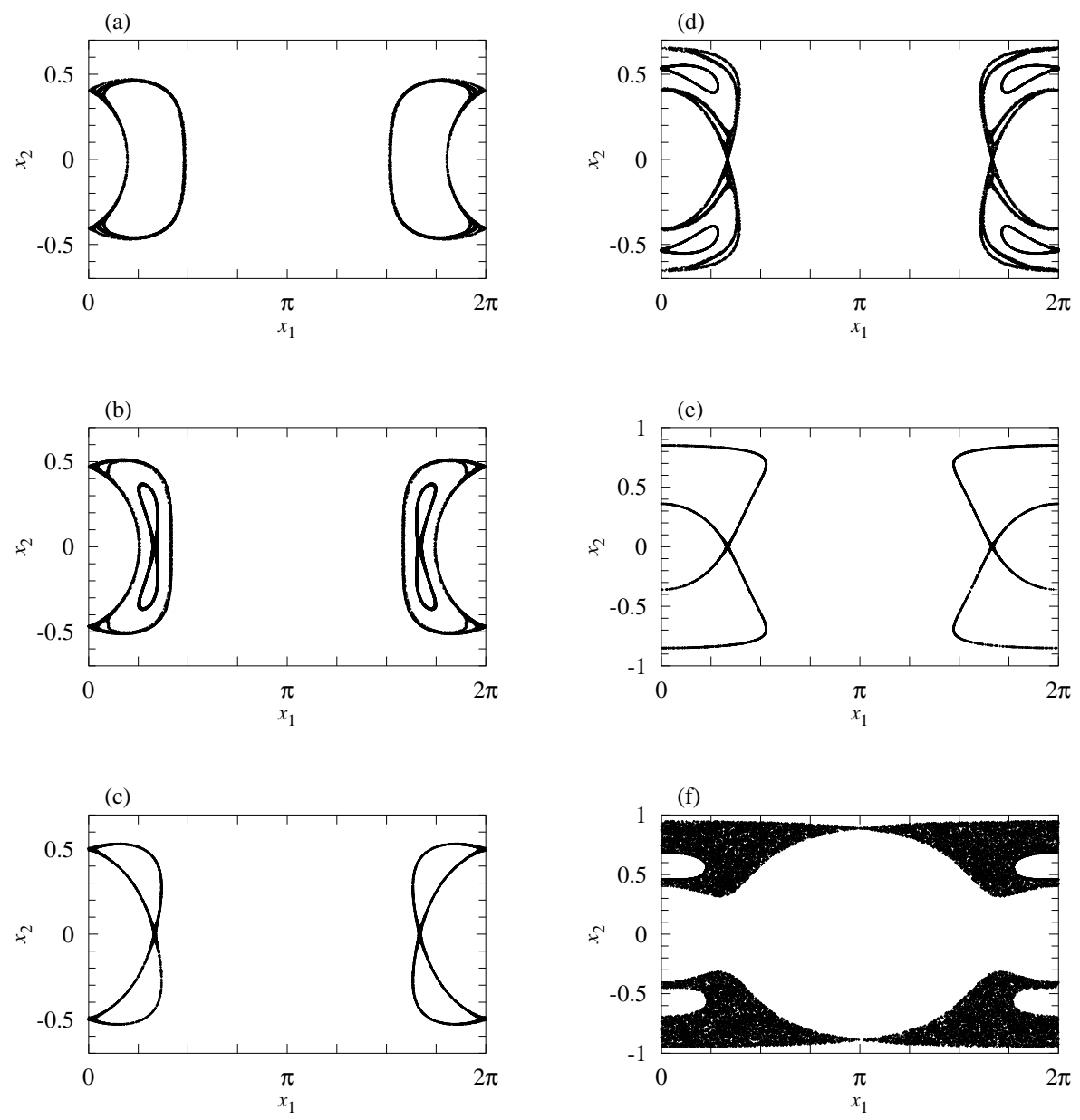

Figure 6: Poincaré sections $\left\{y_{2}=0, \dot{y}_{2}>0\right\}$ for chaotic orbits in $\mathbb{P}_{6}\left(\pi_{\mathrm{e}}\right)$ obtained by direct numerical simulation: (a) $\Gamma=2$; (b) $\Gamma=1.25$; (c) $\Gamma=1$; (d) $\Gamma=0.75$; (e) $\Gamma=0.25$; (f) $\Gamma=-0.1$. More details of data for the chaotic orbits are given in Table 2 .

Fig. $7(\mathrm{~h})$ drifts rotationally on the sphere since the corresponding chaotic orbit of Fig. 6(f) experiences the full range, $0 \leq x_{1} \leq 2 \pi$ as the homoclinic orbits of Fig. 2(f) does.

\section{Detection of chaos in $\mathbb{P}_{5}\left(\pi_{\mathrm{o}}\right)$}

For $\mathbb{P}_{5}\left(\pi_{\mathrm{o}}\right)$, as stated in $\S 3.2$, the analytic technique of $\S 4.1$ is not applicable since it has no invariant plane. Thus, applying only the numerical approach of $\S 4.3$, we compute the stable and unstable manifolds of periodic orbits near the saddle-center and detect the occurrence of chaos.

The Hamiltonian (3.19) with $n=1$ has an equilibrium at $\left(Q_{3}, Q_{4}, P_{3}, P_{4}\right)=$ 
Table 2: Data of the direct numerical simulations of chaotic motions in $\mathbb{P}_{6}\left(\pi_{\mathrm{e}}\right)$. The second and third columns, respectively, give saddle-centers (i) which are closest to the initial points and (ii) which the chaotic orbits pass near homoclinic or heteroclinic orbits to. $H_{0}$ represents the Hamiltonian energy of the saddlecenter of type (i).

\begin{tabular}{c|c|c|c|c|c|c}
\hline \hline$\Gamma$ & (i) & (ii) & $H$ & $H_{0}$ & Fig. 6 & Fig. 7 \\
\hline 2 & $\boldsymbol{s}_{5}$ & $\boldsymbol{s}_{5,6}$ & 0.0995 & 0.09930 & (a) & (a) \\
1.25 & $\boldsymbol{s}_{1}$ & $\boldsymbol{s}_{1,2}$ & 0.03 & 0.02704 & (b) & (b) \\
& $\boldsymbol{s}_{5}$ & $\boldsymbol{s}_{5,6}$ & 0.0485 & 0.04714 & & (c) \\
1 & $\boldsymbol{s}_{5}$ & $\boldsymbol{s}_{1,2,5,6}$ & 0.0275 & 0.02704 & (c) & (d) \\
0.75 & $\boldsymbol{s}_{1}$ & $\boldsymbol{s}_{1,2}$ & 0.02720425 & 0.02704 & (d) & (e) \\
& $\boldsymbol{s}_{5}$ & $\boldsymbol{s}_{5,6}$ & 0.003 & 0.00231 & & $(\mathrm{f})$ \\
0.25 & $\boldsymbol{s}_{1}$ & $\boldsymbol{s}_{1,2}$ & 0.0273 & 0.02704 & (e) & (g) \\
-0.1 & $\boldsymbol{s}_{3}$ & $\boldsymbol{s}_{3,4}$ & -0.0196 & -0.0197 & (f) & $(\mathrm{h})$ \\
\hline \hline
\end{tabular}

$(2 \pi / 5,12 \pi / 5,0,0)$, which corresponds to the 5 -ring at the equator

$$
\boldsymbol{X}_{o}=\left(\frac{\pi}{2}, \frac{\pi}{2}, \frac{\pi}{2}, \frac{\pi}{2}, \frac{\pi}{2}, 0, \frac{2}{5} \pi, \frac{4}{5} \pi,-\frac{4}{5} \pi,-\frac{2}{5} \pi\right),
$$

as in Proposition 2.2. It becomes a saddle-center equilibrium for $0<\Gamma<1$, since the Hessian of the Hamiltonian $H_{1}^{(5)}$ at this point has the eigenvalues

$$
\pm \frac{\sqrt{83+15 \sqrt{5}-150 \Gamma-\sqrt{(83+15 \sqrt{5})^{2}+300 \Gamma(-51-15 \sqrt{5}+43 \Gamma)}}}{4 \sqrt{5} \pi}
$$

which are purely imaginary for $\Gamma>0$ and real for $\Gamma<0$, and

$$
\pm \frac{\sqrt{83+15 \sqrt{5}-150 \Gamma+\sqrt{(83+15 \sqrt{5})^{2}+300 \Gamma(-51-15 \sqrt{5}+43 \Gamma)}}}{4 \sqrt{5} \pi},
$$

which are purely imaginary for $\Gamma>1$ and real for $\Gamma<1$.

Figure 8 shows periodic orbits near the saddle-center for $\Gamma=0.5$, and Figure 9 shows the stable and unstable manifolds of the outermost periodic orbit in Fig. 8 on the Poincaré section $\left\{P_{4}=0\right\}$. Compared with the results of $\mathbb{P}_{6}\left(\pi_{\mathrm{e}}\right)$ in $\S 5$, the behavior of the invariant manifolds is very complicated. This is due to the fact that there is no invariant plane having a homoclinic orbit in $\mathbb{P}_{5}\left(\pi_{\mathrm{o}}\right)$. Nevertheless, we see that they really intersect transversely (without self-intersections) and hence chaotic dynamics occurs. Figure 10 shows the Poincaré section $\left\{P_{4}=0\right\}$ for a trajectory with an initial point near the saddlecenter and its corresponding orbit of the 5-ring on the sphere, which clearly demonstrate chaotic behavior. 
(a)

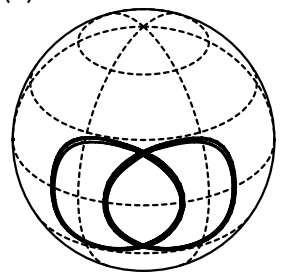

(b)

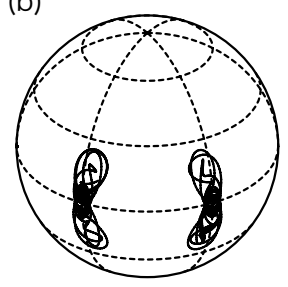

(c)

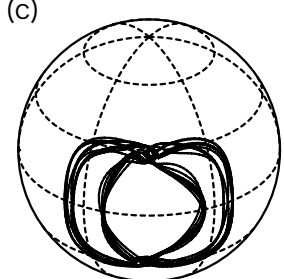

(d)

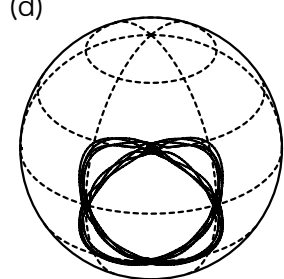

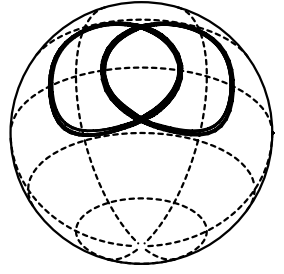
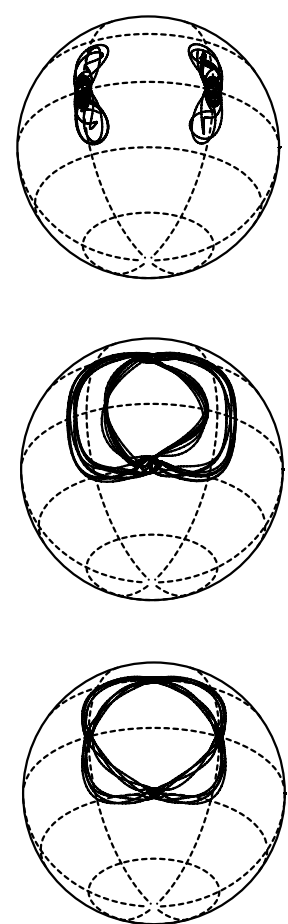

(e)
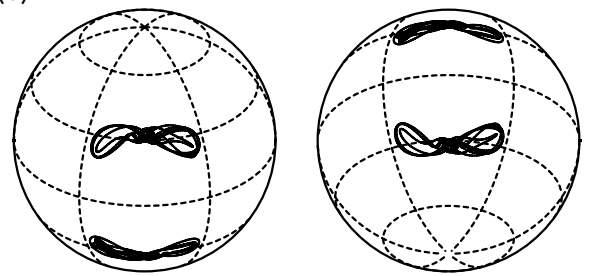

(f)
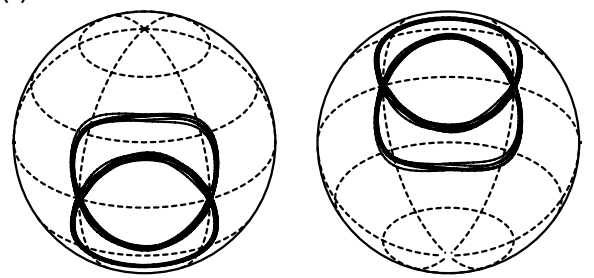

(g)
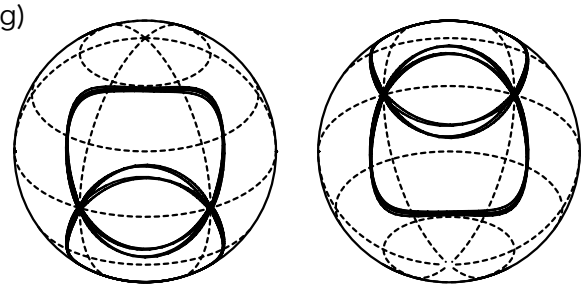

(h)
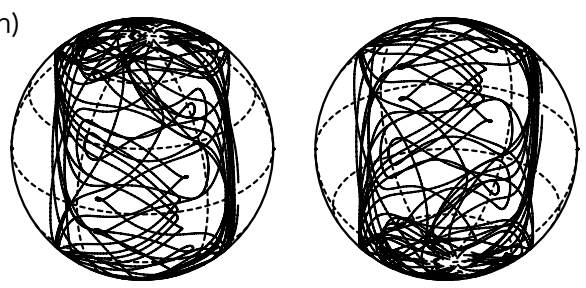

Figure 7: Chaotic trajectories near homo- or heteroclinic orbits to the saddlecenters $\mathbb{P}_{6}\left(\pi_{\mathrm{e}}\right)$, each of which corresponds to the Poincaré section in Fig. 6: (a) $\Gamma=2$; (b) and (c) $\Gamma=1.25$; (d) $\Gamma=1$; (e) and (f) $\Gamma=0.75$; (g) $\Gamma=0.25$; (h) $\Gamma=-0.1$. More details of data for the chaotic trajectories are given in Table 2 . Note that the first and fourth point vortices stay at the equator due to (2.15).

\section{Summary and discussion}

We have shown the existence of chaotic motions in two-degree-of-freedom Hamiltonian systems with saddle-center equilibria derived from the $N$-vortex problem on the sphere according to the reduction method of [39]. Utilizing the analytical and numerical techniques proposed in $[46,49-51]$ for $N=6$, we numerically showed that the stable and unstable manifolds of periodic orbits around saddlecenter equilibria intersect transversely and hence chaotic orbits exist. We also have numerically demonstrated that the unstable and stable manifolds of the periodic orbits near the saddle-center intersect transversely, and given some direct numerical results to illustrate the existence of chaotic orbits for $N=5$ 

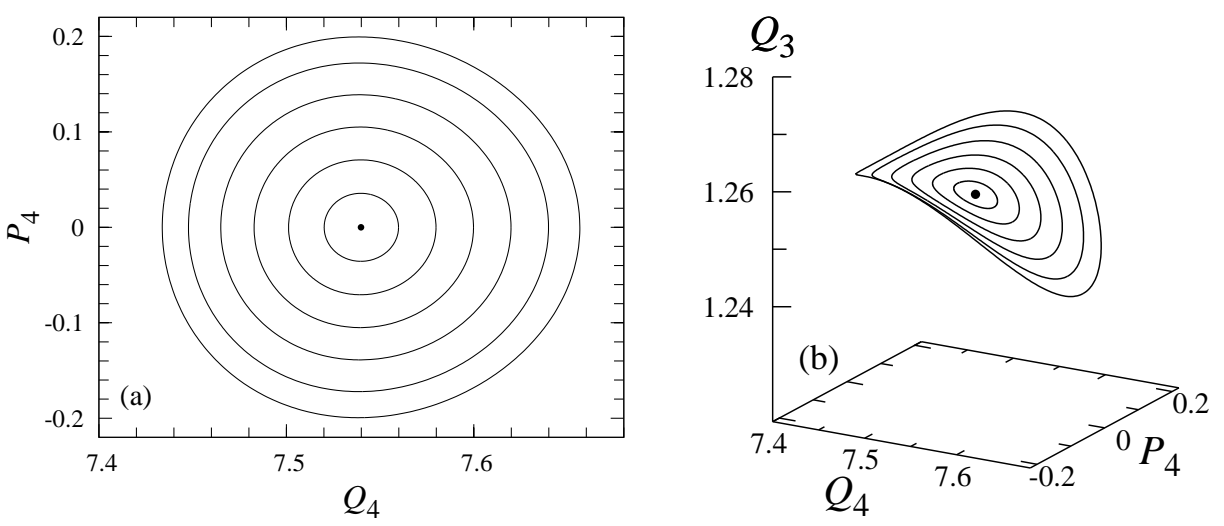

Figure 8: Periodic orbits near the saddle-center $\left(Q_{3}, Q_{4}, P_{3}, P_{4}\right)=$ $(2 \pi / 5,12 \pi / 5,0,0)$ in $\mathbb{P}_{5}\left(\pi_{\mathrm{e}}\right)$ for $\Gamma=0.5$ : (a) Their projections onto the $\left(Q_{4}, P_{4}\right)$ plane; (b) onto the $\left(Q_{4}, P_{4}, Q_{3}\right)$-space.

and 6 .

The analytical and numerical approaches we used depend on the structure of the reduced two-degree-of-freedom system: For $N=6 n$, we make the best use of the Melnikov-type analysis [46] since the reduced system satisfies a key assumption in the analysis, i.e., it contains a two-dimensional invariant plane consisting of the stable and unstable eigenspaces. On the other hand, the Melnikov analysis is not applicable for $N=5 n$ since the reduced system has no such invariant plane. Thus we rely on the direct numerical computation of the unstable and the stable manifolds [51] to demonstrate their transverse intersection.

Here we discuss how chaotic orbits found in the reduced system appear in the full 6-vortex problem on the sphere. We first note that if the 6-ring corresponds to a saddle-center equilibrium in the reduced system $\mathbb{P}_{6}\left(\pi_{\mathrm{e}}\right)$, then $\left(\lambda_{1}^{ \pm}\right)^{2}<0<\left(\lambda_{2}^{ \pm}\right)^{2}$ and hence the maximum eigenvalue $\lambda_{3}^{+}$becomes positive since $\left(\lambda_{2}^{ \pm}\right)^{2}<\left(\lambda_{3}^{ \pm}\right)^{2}$. Thus, the eigenspace for $\lambda_{3}^{+}$, which is not included in the reduced system, give the most unstable directions, and inevitably affects the dynamics of the full system. However, we expect that solutions of the full system also exhibit chaotic motions since by the Poincaré recurrence theorem [1], they must repeatedly return in neighborhoods of chaotic orbits in the reduced system if they start there.

Figure 11 shows numerical solutions of the full system (1.1) and (1.2) from $t=0$ to 800 for the identical pole vortices $\Gamma_{1,2}=\Gamma=1.25$ or 1 , where the DOP853 code [14] is also used with a tolerance of $10^{-10}$. Their initial conditions are taken near the 6-ring at the equator as

$$
\boldsymbol{X}(0)=\boldsymbol{X}_{\mathrm{e}}+\varepsilon_{1} \frac{\boldsymbol{\phi}_{1}^{+}+\boldsymbol{\phi}_{1}^{-}}{\left|\boldsymbol{\phi}_{1}^{+}+\boldsymbol{\phi}_{1}^{-}\right|}+\varepsilon_{2} \frac{\boldsymbol{\psi}_{1}^{+}+\boldsymbol{\psi}_{1}^{-}}{\left|\boldsymbol{\psi}_{1}^{+}+\boldsymbol{\psi}_{1}^{-}\right|},
$$

where $\left(\varepsilon_{1}, \varepsilon_{2}\right)=\left(10^{-3}, 10^{-6}\right)$ for $\Gamma=1.25$ and $\left(10^{-4}, 10^{-7}\right)$ for $\Gamma=1$. By $(2.13)$, the first disturbance in (7.1) is included in the invariant space $\mathbb{P}_{6}\left(\pi_{\mathrm{e}}\right)$, while the 

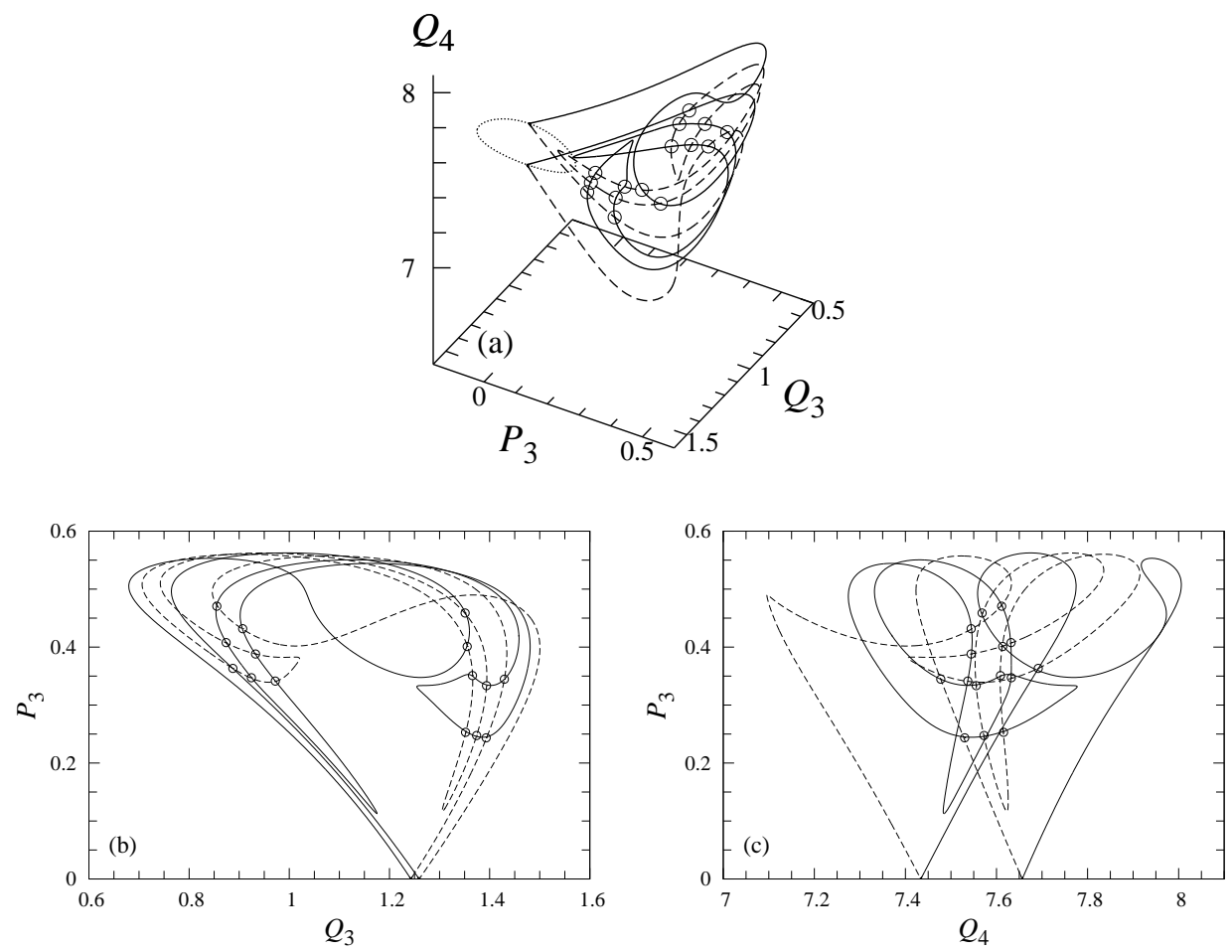

Figure 9: Stable and unstable manifolds of a periodic orbit near the saddlecenter $\left(Q_{3}, Q_{4}, P_{3}, P_{4}\right)=(2 / 5 \pi, 12 / 5 \pi, 0,0)$ in $\mathbb{P}_{5}\left(\pi_{\mathrm{e}}\right)$ on the Poincaré section $\left\{P_{4}=0\right\}$ for $\Gamma=0.5$ and $H=-0.087$; (a) In the $\left(Q_{3}, P_{3}, Q_{4}\right)$-space; (b) their projections onto the $\left(Q_{3}, P_{3}\right)$-plane; (c) onto the $\left(Q_{4}, P_{3}\right)$-plane. The solid and broken lines represent the stable and unstable manifolds, respectively, and the circle "o" represents homoclinic points. The dotted lines represents the projection of the periodic orbit (not on the Poincaré section) onto the $\left(Q_{3}, P_{3}, Q_{4}\right)$-space in plate (a).

second one is in its complementary space. The trajectories in Figs. 11(a) and (b) seem to move around the chaotic ones of the reduced system in Figs. 7(b) and (d), respectively. Figure 12 displays how far the trajectories of the full system travel from the invariant space $\mathbb{P}_{6}\left(\pi_{\mathrm{e}}\right)$, where their distance from $\mathbb{P}_{6}\left(\pi_{\mathrm{e}}\right)$ is estimated by

$$
\begin{aligned}
L^{2}= & P_{1}^{2}+P_{2}^{2}+\left(P_{3}-P_{6}\right)^{2}+\left(P_{4}-P_{5}\right)^{2} \\
& +\sin ^{2} \frac{Q_{1}}{2}+\sin ^{2} \frac{Q_{3}-Q_{6}}{2}+\sin ^{2} \frac{Q_{4}-Q_{5}}{2}+\sin ^{2} \frac{Q_{2}+Q_{3}+Q_{4}-\pi}{2}
\end{aligned}
$$

Note that $L \approx 0$ when the trajectories travel near $\mathbb{P}_{6}\left(\pi_{\mathrm{e}}\right)$, and actually $L=0$ when they do in $\mathbb{P}_{6}\left(\pi_{\mathrm{e}}\right)$, due to (3.11) and (3.14). From Fig. 12 we see that the trajectories sometimes approach to $\mathbb{P}_{6}\left(\pi_{\mathrm{e}}\right)$ but they immediately get far away from it after that. We tried to find such orbits as predicted by the Poincaré recurrence theorem, but we could not succeed since they require very long time to return to a small neighborhood of $\mathbb{P}_{6}\left(\pi_{\mathrm{e}}\right)$, say with a width of $O\left(\varepsilon_{2}\right)$, and it 


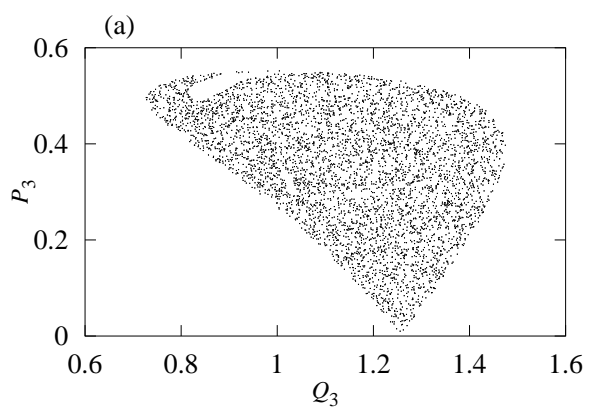

(b)

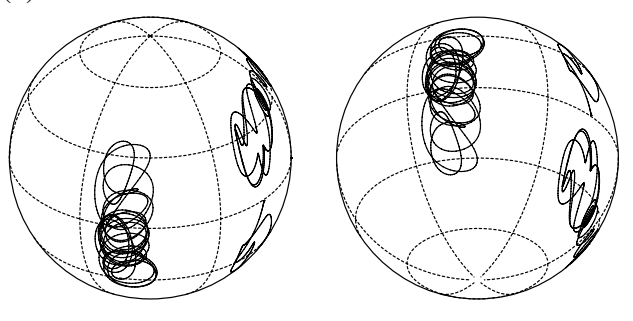

Figure 10: (a) Poincaré section $\left\{P_{4}=0\right\}$ for a chaotic motion in $\mathbb{P}_{5}\left(\pi_{\mathrm{e}}\right)$ with an initial point near the saddle center $\left(Q_{3}, Q_{4}, P_{3}, P_{4}\right)=(2 / 5 \pi, 12 / 5 \pi, 0,0)$ for $\Gamma=0.5$; (b) its corresponding chaotic orbit near homoclinic orbits to the 5 -ring on the sphere. We see the only four trajectories on the sphere, since the first point vortex in $\mathbb{P}_{5}\left(\pi_{\mathrm{o}}\right)$ is fixed at the equator due to $(2.14)$.

(a)

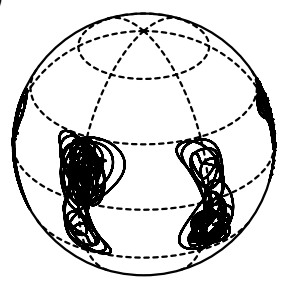

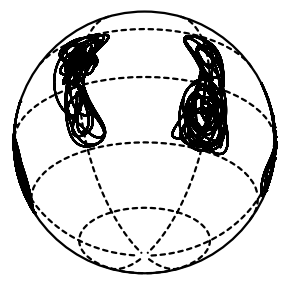

(b)

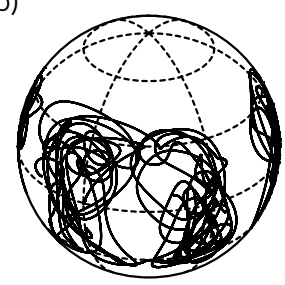

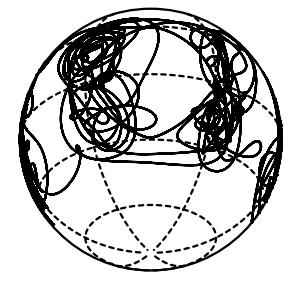

Figure 11: Chaotic trajectories of the perturbed 6-ring in the full 6-vortex problem (1.1) and (1.2) up to $t=800$ in the presence of the identical pole vortices: (a) $\Gamma=1.25$; (b) $\Gamma=1$.

is very hard to track solutions of the full system numerically with high accuracy for such long time even though a highly efficient, numerical integrator like the DOP853 code is used. In Fig. 13 we plot the time histories of the $P_{3}$-component for the same initial conditions and slightly different ones, $\varepsilon_{1}=2 \times 10^{-3}$ for $\Gamma=1.25$ and $\varepsilon_{1}=5 \times 10^{-4}$ for $\Gamma=1$ with the same value of $\varepsilon_{2}$. As shown in Fig. 13, the trajectories depend sensitively on initial conditions, and they are chaotic in this sense. Moreover, in Figs. 12 and 13 we observe that they separate away while they still remain near $\mathbb{P}_{6}\left(\pi_{\mathrm{e}}\right)$, although their initial conditions are different only in a direction of $\mathbb{P}_{6}\left(\pi_{\mathrm{e}}\right)$. Thus, the instability of motions in the directions of $\mathbb{P}_{6}\left(\pi_{\mathrm{e}}\right)$ is also revealed.

Finally we comment on further research for $N \neq 5 n, 6 n$ with $n \in \mathbb{N}$. The Melnikov-type method was extended to three- or more-degree-of-freedom Hamiltonian systems with saddle-centers in [47]. The reduced systems $\mathbb{P}_{N}\left(\pi_{\mathrm{o}}\right)$ and $\mathbb{P}_{N}\left(\pi_{\mathrm{e}}\right)$ in (2.12) and (2.13) are multi-degree-of-freedom Hamiltonian and the $N$-ring corresponds to a saddle-center equilibrium for a certain range of $\Gamma$. So the reader may think that it is also applicable to the $N$ vortex problem on the sphere but this is not the case. For instance, Proposition 2.3 shows that $\mathbb{P}_{8}\left(\pi_{\mathrm{e}}\right)$ 
(a) $\Gamma=1.25$

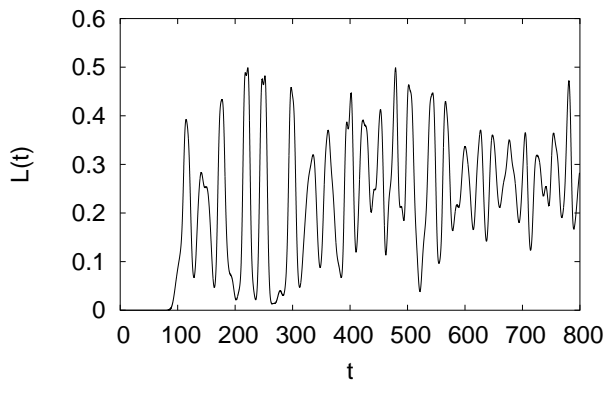

(b) $\Gamma=1.00$

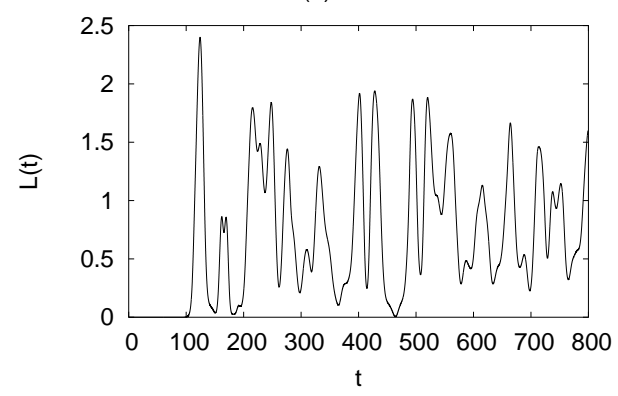

Figure 12: Distance of the trajectories of Fig. 11 from the invariant space $\mathbb{P}_{6}\left(\pi_{\mathrm{e}}\right)$ in the full 6-vortex problem: (a) $\Gamma=1.25$; (b) $\Gamma=1$.

(a) $\Gamma=1.25$

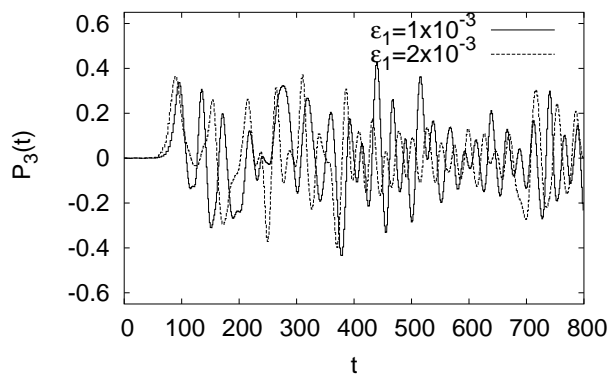

(b) $\Gamma=1.00$

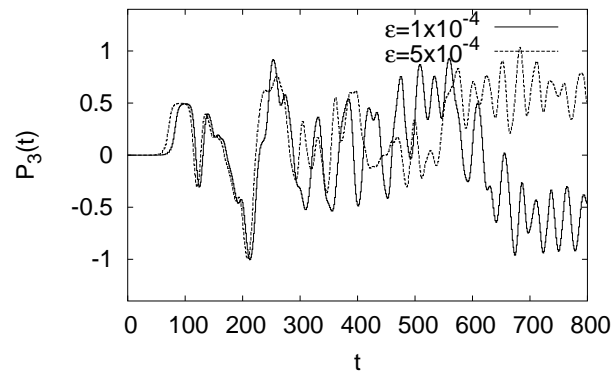

Figure 13: Sensitive dependence on initial conditions in the full 6 -vortex problem: (a) $\Gamma=1.25$; (b) $\Gamma=1$. The time histories of the $P_{3}$-component are plotted for two initial conditions such that $\varepsilon_{1}=10^{-3}$ and $2 \times 10^{-3}$ with $\varepsilon_{2}=10^{-6}$ in plate (a), and $\varepsilon_{1}=10^{-4}$ and $5 \times 10^{-4}$ with $\varepsilon_{2}=10^{-7}$ in plate (b).

is a multi-degree-of-freedom Hamiltonian system in which the phase space is represented by

$$
\boldsymbol{X}=\boldsymbol{X}_{\mathrm{e}}+\sum_{k=1}^{3}\left(b_{k}^{+} \boldsymbol{\phi}_{k}^{+}+b_{k}^{-} \boldsymbol{\phi}_{k}^{-}\right), \quad b_{k}^{ \pm} \in \mathbb{R},
$$

where $\boldsymbol{X}_{\mathrm{e}}$ represents the 8-ring at the equator. The 8-ring becomes a saddlecenter equilibrium when the eigenvalues $\lambda_{3}^{+}$and $\lambda_{3}^{-}$are positive and negative, respectively, and the other eigenvalues $\lambda_{2}^{ \pm}$and $\lambda_{1}^{ \pm}$are purely imaginary, but the eigenspace spanned by the eigenvectors for $\lambda_{3}^{ \pm}$is not invariant for $\mathbb{P}_{8}\left(\pi_{\mathrm{e}}\right)$. (See the list of invariant dynamical systems in $\mathbb{P}_{8}$ in [40].) Thus the Melnikov-type method of [47] is not applicable. However, the numerical approach given in $\S 4.3$ is still available and numerical results in this direction will be reported in [42]. 


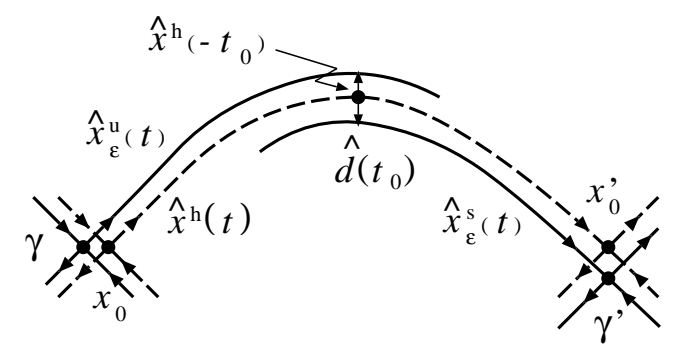

Figure A1: Orbits on the stable and unstable manifolds

\section{A Proof of Theorem 4.2}

We outline the proof of Theorem 4.2, which is very similar to that of Theorem 1.1 of [46].

Let $\hat{\boldsymbol{z}}_{\varepsilon}^{\mathrm{u}}\left(t ; t_{0}\right)=\left(\hat{\boldsymbol{x}}_{\varepsilon}^{\mathrm{u}}\left(t ; t_{0}\right), \hat{\boldsymbol{y}}_{\varepsilon}^{\mathrm{u}}\left(t ; t_{0}\right)\right)$ and $\hat{\boldsymbol{z}}_{\varepsilon}^{\mathrm{s}}\left(t ; t_{0}\right)=\left(\hat{\boldsymbol{x}}_{\varepsilon}^{\mathrm{s}}\left(t ; t_{0}\right), \hat{\boldsymbol{y}}_{\varepsilon}^{\mathrm{s}}\left(t ; t_{0}\right)\right)$ be orbits on the unstable manifold of a periodic orbit $\gamma$ near the saddle-center $\left(\boldsymbol{x}_{0}^{\prime}, \mathbf{0}\right)$ and on the stable manifold of a periodic orbit $\gamma^{\prime}$ near the saddle-center $\left(\boldsymbol{x}_{0}, \mathbf{0}\right)$, respectively, such that they pass near $(\boldsymbol{x}, \boldsymbol{y})=\left(\hat{\boldsymbol{x}}^{\mathrm{h}}\left(-t_{0}\right), \mathbf{0}\right)$ and the distances between $\gamma$ and $\left(\boldsymbol{x}_{0}, \mathbf{0}\right)$ and between $\gamma^{\prime}$ and $\left(\boldsymbol{x}_{0}^{\prime}, \mathbf{0}\right)$ are $O(\varepsilon)$, where $\varepsilon$ is a constant such that $0<\varepsilon \ll 1$. See Fig. A1. As in Lemma 3.2 of [46], we can represent the orbits $\boldsymbol{z}_{\varepsilon}^{\mathrm{s}, \mathrm{u}}\left(t ; t_{0}\right)$ as

$$
\hat{\boldsymbol{x}}_{\varepsilon}^{\mathrm{u}}\left(t ; t_{0}\right)=\hat{\boldsymbol{x}}^{\mathrm{h}}\left(t-t_{0}\right)+O\left(\varepsilon^{2}\right), \quad \hat{\boldsymbol{y}}_{\varepsilon}^{\mathrm{u}}\left(t ; t_{0}\right)=\varepsilon \hat{\Psi}\left(t-t_{0}\right) \hat{B}_{-}^{-1} \Phi\left(t_{0}\right) \boldsymbol{\eta}_{0}
$$

for $t \in\left[-t_{2}, 0\right]$ and

$$
\hat{\boldsymbol{x}}_{\varepsilon}^{\mathrm{s}}\left(t ; t_{0}\right)=\hat{\boldsymbol{x}}^{\mathrm{h}}\left(t-t_{0}\right)+O\left(\varepsilon^{2}\right), \quad \hat{\boldsymbol{y}}_{\varepsilon}^{\mathrm{s}}\left(t ; t_{0}\right)=\varepsilon \hat{\Psi}\left(t-t_{0}\right) \hat{B}_{+}^{-1} \hat{\Phi}\left(t_{0}\right) \boldsymbol{\eta}_{0}
$$

for $t \in\left[0, t_{1}\right]$, where $t_{1}$ and $t_{2}$ are any positive numbers. Let $d\left(t_{0} ; \boldsymbol{\eta}_{0}, \varepsilon\right)$ be the distance between the orbits $\hat{\boldsymbol{z}}_{\varepsilon}^{\mathrm{s}, \mathrm{u}}\left(t ; t_{0}\right)$ along the direction $\left(\mathrm{D}_{x} H\left(\hat{\boldsymbol{x}}^{\mathrm{h}}\left(-t_{0}\right), \mathbf{0}\right), \mathbf{0}\right)$ at $t=0$ (see Fig. A1). Using an argument given in $\S 3$ of [46], we can show that

$$
\frac{1}{\varepsilon^{2}} d\left(t_{0} ; \boldsymbol{\eta}_{0}, \varepsilon\right)=\frac{\tilde{M}\left(t_{0}\right)}{\left|\mathrm{D}_{x} H\left(\hat{\boldsymbol{x}}^{\mathrm{h}}\left(-t_{0}\right), \mathbf{0}\right)\right|}+O(\varepsilon),
$$

where

$$
\tilde{M}\left(t_{0}\right)=\int_{-\infty}^{\infty} \mathrm{D}_{x} H\left(\hat{\boldsymbol{x}}^{\mathrm{h}}(t), \mathbf{0}\right) \cdot \boldsymbol{g}\left(\hat{\boldsymbol{x}}^{\mathrm{h}}(t), \hat{\Psi}(t) \hat{B}_{-}^{-1} \Phi\left(t_{0}\right) \boldsymbol{\eta}_{0}\right) \mathrm{d} t
$$

with

$$
\boldsymbol{g}(\boldsymbol{x}, \boldsymbol{\eta})=\frac{1}{2} \mathrm{D}_{y}^{2} \boldsymbol{f}(\boldsymbol{x}, \mathbf{0})(\boldsymbol{\eta}, \boldsymbol{\eta}), \quad \boldsymbol{f}(\boldsymbol{x}, \boldsymbol{y})=J_{1} \mathrm{D}_{x} H(\boldsymbol{x}, \boldsymbol{y}) .
$$

We easily prove the following lemma in a manner analogous to Lemmas 3.3 and 3.4 of [46].

Lemma A.1. For any $\boldsymbol{\eta}_{0}, \boldsymbol{\eta}_{0}^{\prime}(\neq 0) \in \mathbb{R}^{2}$ and $t_{0}^{\prime} \in \mathbb{R}$, there exist real numbers $\rho, t_{0}$ such that

$$
\tilde{M}\left(t_{0}^{\prime} ; \boldsymbol{\eta}_{0}^{\prime}\right)=\rho^{2} \tilde{M}\left(t_{0} ; \boldsymbol{\eta}_{0}\right) \text {. }
$$


Let $\boldsymbol{\eta}(t)=\hat{\Psi}(t) \hat{\boldsymbol{\eta}}_{0}$ for any $\hat{\boldsymbol{\eta}}_{0} \in \mathbb{R}^{2}$. As in the proof of Lemma 3.4 of [46], we have

$$
\frac{\mathrm{d}}{\mathrm{d} t}\left[\mathrm{D}_{y}^{2} H\left(\boldsymbol{x}^{\mathrm{h}}(t), \mathbf{0}\right)(\boldsymbol{\eta}(t), \boldsymbol{\eta}(t))\right]=-2 \mathrm{D}_{x} H\left(\boldsymbol{x}^{\mathrm{h}}(t), \mathbf{0}\right) \cdot \boldsymbol{g}\left(\boldsymbol{x}^{\mathrm{h}}(t), \boldsymbol{\eta}(t)\right) .
$$

Hence

$$
\int_{-\infty}^{\infty} \mathrm{D}_{x} H\left(\hat{\boldsymbol{x}}^{\mathrm{h}}(t), \mathbf{0}\right) \cdot \boldsymbol{g}\left(\boldsymbol{x}^{\mathrm{h}}(t), \boldsymbol{\eta}(t)\right) \mathrm{d} t=m(\boldsymbol{\eta}(-\infty))-m^{\prime}(\boldsymbol{\eta}(\infty)) .
$$

Substituting $\boldsymbol{\eta}(t)=\hat{\Psi}(t) \hat{B}_{-}^{-1} \Phi\left(t_{0}\right) \boldsymbol{\eta}_{0}$ into (A.6) and using (4.8), we prove the following lemma.

Lemma A.2. We have

$$
\tilde{M}_{1}\left(t_{0} ; \boldsymbol{\eta}_{0}\right)=m\left(\boldsymbol{\eta}_{0}\right)-m^{\prime}\left(\hat{B}_{0} \Phi\left(t_{0}\right) \boldsymbol{\eta}_{0}\right) .
$$

In particular, for $\boldsymbol{e}_{1}=(1,0)^{\mathrm{T}}$

$$
\tilde{M}_{1}\left(t_{0} ; \boldsymbol{e}_{1}\right)=\hat{M}\left(t_{0}\right) .
$$

From Lemmas A.1 and A.2 we see that if $\hat{M}\left(t_{0}\right)$ has a simple zero, then $\tilde{M}\left(t_{0} ; \boldsymbol{\eta}_{0}\right)$ does so for any nonzero $\boldsymbol{\eta}_{0} \in \mathbb{R}^{2}$. Applying the implicit function theorem to (A.3) at $\varepsilon=0$, we complete the proof of Theorem 4.2.

\section{B Numerical analyses of $\S \S 4.2$ and 4.3 for the Hénon- Heiles system}

We perform the numerical analyses of $\S \S 4.2$ and 4.3 for the generalized HénonHeiles system, for which some analytical results are available $[12,46,48]$ in order to demonstrate their accuracy.

\section{B.1 Analytical results}

The generalized Hénon-Heiles system is given by

$$
\begin{aligned}
& \dot{x}_{1}=x_{2}, \quad \dot{x}_{2}=-x_{1}-c y_{1}^{2}-d x_{1}^{2}, \\
& \dot{y}_{1}=y_{2}, \quad \dot{y}_{2}=-y_{1}-2 c x_{1} y_{2},
\end{aligned}
$$

where $c, d$ are positive constants. In the original one of Hénon and Heiles [16] $c=1$ and $d=-1$. The system (B.1) is integrable for $c / d=0,1 / 6,1$ (see, e.g., Ref. [11]). There is a saddle-center at

$$
\left(x_{1}, x_{2}, y_{1}, y_{2}\right)=(-1 / 2 c, 0, \pm \sqrt{2-(d / c)} / 2 c, 0)
$$

if $c / d>1 / 2$, and at $(1 / d, 0,0,0)$ if $c / d<1 / 2$. Moreover, when $c / d>1 / 2$ (resp. $c / d<1 / 2)$, it is transformed to

$$
\begin{aligned}
& \dot{x}_{1}=x_{2}, \quad \dot{x}_{2}=x_{1}-a_{1} x_{1}^{2}-\frac{1}{2} a_{2} y_{1}^{2}, \\
& \dot{y}_{1}=y_{2}, \quad \dot{y}_{2}=-\omega^{2} y_{1}-a_{2} x_{1} y_{1}-a_{3} y_{1}^{2}
\end{aligned}
$$


with the Hamiltonian

$$
H(x, y)=\frac{1}{2}\left(x_{2}^{2}+y_{2}^{2}\right)+\frac{1}{2}\left(-x_{1}^{2}+\omega^{2} y_{1}^{2}\right)+\frac{a_{1}}{3} x_{1}^{3}+\frac{a_{2}}{2} x_{1} y_{1}^{2}+\frac{a_{3}}{3} y_{1}^{3},
$$

by a change of coordinates

$$
\begin{aligned}
& x_{1} \mapsto \frac{x_{1}-x_{10}+\mu y_{1}}{\sqrt{1+\mu^{2}}}, \quad x_{2} \mapsto \frac{x_{2}+\mu y_{2}}{\sqrt{1+\mu^{2}}}, \\
& y_{1} \mapsto \frac{-\mu\left(x_{1}-x_{10}\right)+y_{1}}{\sqrt{1+\mu^{2}}}, \quad y_{2} \mapsto \frac{-\mu x_{2}+y_{2}}{\sqrt{1+\mu^{2}}}
\end{aligned}
$$

(resp. $x_{1} \mapsto x_{1}-1 / d$ ), where

$$
\begin{aligned}
& a_{1}=\frac{2 c}{\sqrt{1+\mu^{2}}}, \quad a_{2}=\frac{2(d-c)}{\sqrt{1+\mu^{2}}}, \quad a_{3}=\frac{\mu(c+d)}{\sqrt{1+\mu^{2}}}, \\
& \omega=\frac{\mu}{\sqrt{2}}, \quad x_{10}=\frac{\sqrt{1+\mu^{2}}}{2 c}, \quad \mu=\sqrt{2-\frac{d}{c}}
\end{aligned}
$$

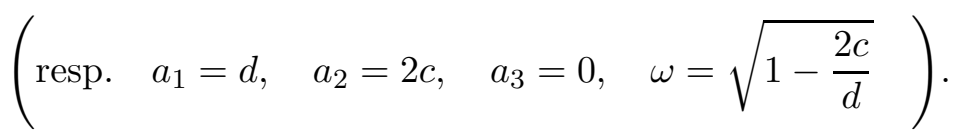

The transformed system (B.2) satisfies assumptions (A1)-(A3) in $§ 4.1$. In particular, the origin is a saddle-center and has a homoclinic orbit given by

$$
x^{\mathrm{h}}(t)=\left(\frac{3}{2 a_{1}} \operatorname{sech}^{2}\left(\frac{t}{2}\right),-\frac{3}{2 a_{1}} \operatorname{sech}^{2}\left(\frac{t}{2}\right) \tanh \left(\frac{t}{2}\right)\right)
$$

on the $\boldsymbol{x}$-plane. The components of the Hessian matrix of the Hamiltonian at the origin in (4.11) are given by

$$
m_{1}=\omega^{2}, \quad m_{2}=1 .
$$

We compute the Melnikov function (4.6) as

$$
M\left(t_{0}\right)=\omega^{2} b\left(\sqrt{1+b^{2}} \cos \left(2 \omega t_{0}+\tau\right)-b\right),
$$

where $\tau$ is a constant and

$$
b= \begin{cases}\left|\frac{\cos (\pi \sqrt{\sigma} / 2)}{\sinh 2 \pi \omega}\right| & \text { if } \sigma>0 \\ \left|\frac{\cosh (\pi \sqrt{-\sigma} / 2)}{\sinh 2 \pi \omega}\right| & \text { if } \sigma<0\end{cases}
$$

with $\sigma=24\left(a_{2} / a_{1}\right)+1$. See $\S 6$ of [46] for the derivation of (B.4). Thus, we have

$$
\Delta=\frac{m_{2}}{m_{1}}\left[\left(2 \omega^{2} b \sqrt{1+b^{2}}\right)^{2}-\left(2 \omega^{2} b^{2}\right)^{2}\right]=4 \omega^{2} b^{2}
$$

and prove rigorously that chaotic orbits exist in (B.2) if

$$
\frac{a_{2}}{a_{1}} \neq \frac{l(l-1)}{6}, \quad l \in \mathbb{N} .
$$

Note that the criterion (B.6) is independent of $\omega$ and $a_{3}$. The same criterion was also obtained by another approach in [12]. 

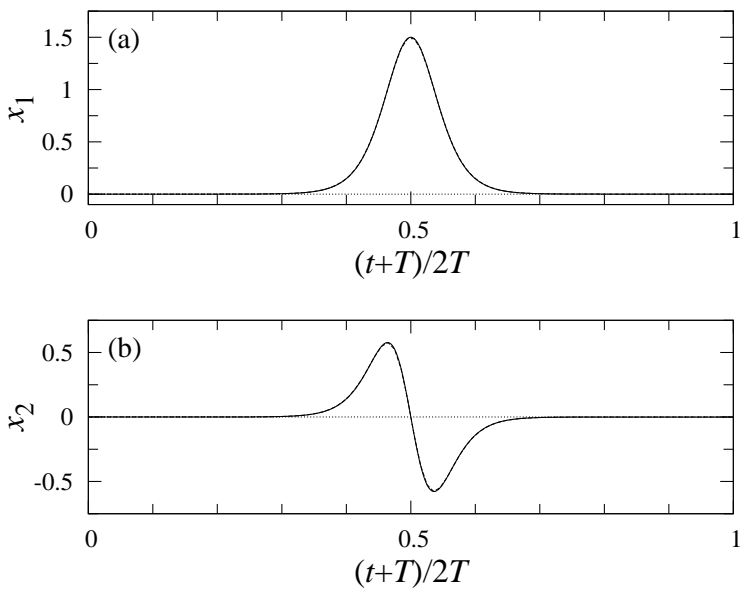

Figure B1: Numerically computed homoclinic orbit in (B.7) for $a_{1}=1$ and $2 T=36.512872$. The analytical expressions (B.3) are also plotted as dashed lines in both plates but almost completely coincide with the numerical results.

\section{B.2 Numerical Melnikov analysis}

Now we apply the numerical method of $\S 4.2$ to (B.2). We first numerically solve the restriction of (B.2) on the $\boldsymbol{x}$-plane,

$$
\dot{x}_{1}=x_{2}, \quad \dot{x}_{2}=x_{1}-a_{1} x_{1}^{2},
$$

as an initial value problem using the approach described in $\S 5$ for the 6 -vortex problem, to compute the homoclinic orbit, which is analytically expressed by (B.3). Figure B1 shows the numerical result for $a_{1}=1$ and $2 T=36.512872$. Here the initial condition $\boldsymbol{x}(-T)$ and the time $T$ are chosen such that $|\boldsymbol{x}(-T)|=$ $10^{-7}$ and $|\boldsymbol{x}(T)| \approx 10^{-7}$. We see that the homoclinic orbit is computed very accurately.

We next numerically compute the criterion (4.21) for the existence of transverse homoclinic orbits, according to the recipe of $\S 4.2$. The NVE (4.4) along the homoclinic orbit becomes

$$
\dot{\eta}_{1}=\eta_{2}, \quad \dot{\eta}_{2}=-\left(\omega^{2}+a_{2} x_{1}^{\mathrm{h}}(t)\right) \eta_{1},
$$

which is solved numerically along with (B.7) in application of the method, where $x_{1}^{\mathrm{h}}(t)$ is numerically obtained. Figure B2 shows the numerical computation of $\Delta$ for $a_{2}=\omega=0.5$ when $a_{1}$ is varied. The numerical result almost completely agrees with the analytical one given by (B.5), which is also plotted in the figure. In particular, we see that $\Delta=0$ when $a_{1} \approx 1.5,0.5,0.25,0.15,0.1$ as indicated in (B.6). Thus, our numerical computation of $\Delta$ is found very accurate.

\section{B.3 Numerical computation of stable and unstable manifolds}

Finally we apply the numerical method of $\S 4.3$ to (B.2). We first note that condition (B.6) is sufficient but not necessary for transverse intersection between 


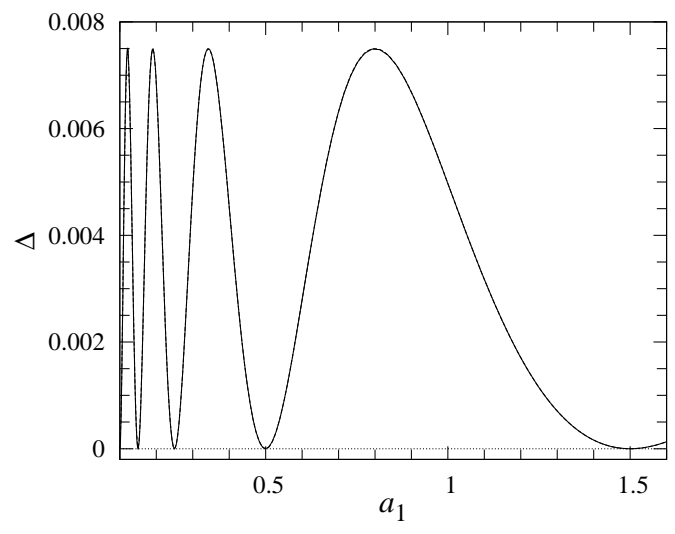

Figure B2: Numerical computations of $\Delta$ for (B.2) with $\omega, a_{2}=0.5$. The analytical expression (B.5) is also plotted as a broken line although its difference from the numerical result is invisible.

the stable and unstable manifolds of periodic orbits near the origin. Actually, it was proved by the higher-order Melnikov-type technique in [48] that such transverse intersection still occurs when $a_{2} / a_{1}=1 / 3$ and $a_{3} \neq 0$.

We choose two sets of parameters for our numerical computation:

$$
\begin{aligned}
& \text { (i) } a_{1}=\frac{3}{2} \sqrt{\frac{3}{5}}, a_{2}=\frac{1}{2} \sqrt{\frac{3}{5}}, a_{3}=\frac{7}{4} \sqrt{\frac{2}{5}} \text { and } \omega=\sqrt{\frac{1}{3}} \\
& \text { (ii) } a_{1}=3, a_{2}=1, a_{3}=0 \text { and } \omega=\sqrt{\frac{2}{3}}
\end{aligned}
$$

Cases (i) and (ii) correspond to $(c, d)=(0.75,1)$ and $(0.5,3)$, respectively, in (B.1). In case (i) $a_{2} / a_{1}=1 / 3$ and $a_{3} \neq 0$ so that condition (B.6) does not hold but the stable and unstable manifolds intersect transversely although their splitting distance is very small [48], while in case (ii) $c / d=1 / 6$ so that the system (B.1) is integrable and such transverse intersection never occurs, as stated above.

Figure B3 shows periodic orbits near the origin in (B.2) for cases (i) and (ii), and Figure B4 does the stable and unstable manifolds of the outermost periodic orbits of Fig. B3 with $H=0.005$ on the Poincaré section $\left\{y_{1}=0\right\}$. Here we set $\varepsilon_{\mathrm{s}}=\varepsilon_{\mathrm{u}}=10^{-4}$ through the computation of Fig. B4, and $\varepsilon_{\mathrm{c}}=10^{-6}$ and $T_{\mathrm{u}}=$ $T_{\mathrm{s}}=10^{-2}$ for the starting solutions, as stated in $\S 4.3$. We see that the stable and unstable manifolds intersect transversely in Fig. B4(a) for case (i), while they almost completely coincide and their difference is invisible in Fig. B4(b) for case (ii). Thus, the numerical method of $\S 4.3$ correctly detects the existence of transverse homoclinic orbits even when the Melnikov-type technique of $\S 4.1$ misses it, and it is admitted very reliable. 

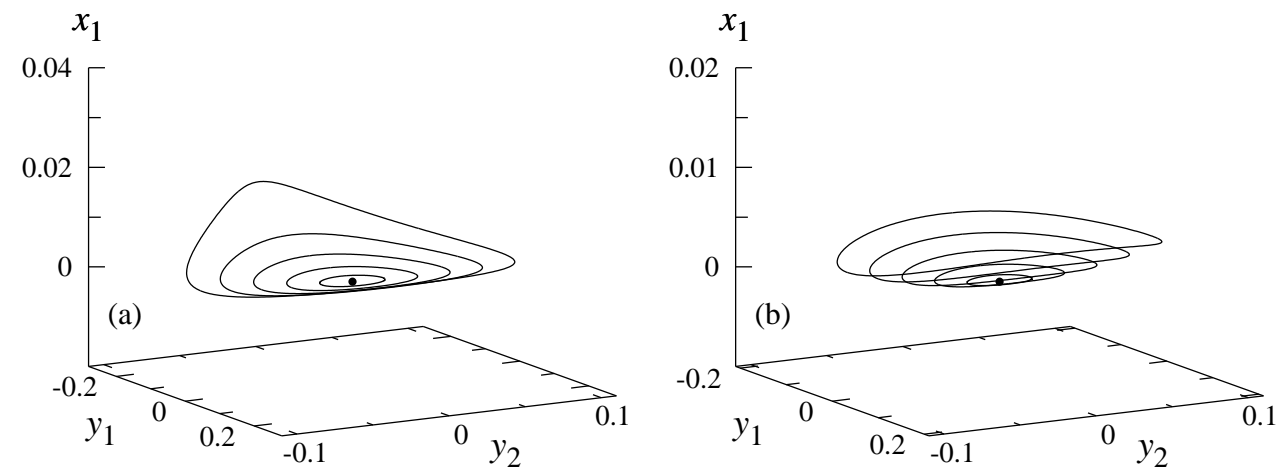

Figure B3: Periodic orbits near the origin in (B.2): (a) Case (i); (b) case (ii). Their projections to the $\left(y_{1}, y_{2}, x_{1}\right)$-space are plotted. The outermost periodic orbit has Hamiltonian energy of $H=0.005$.
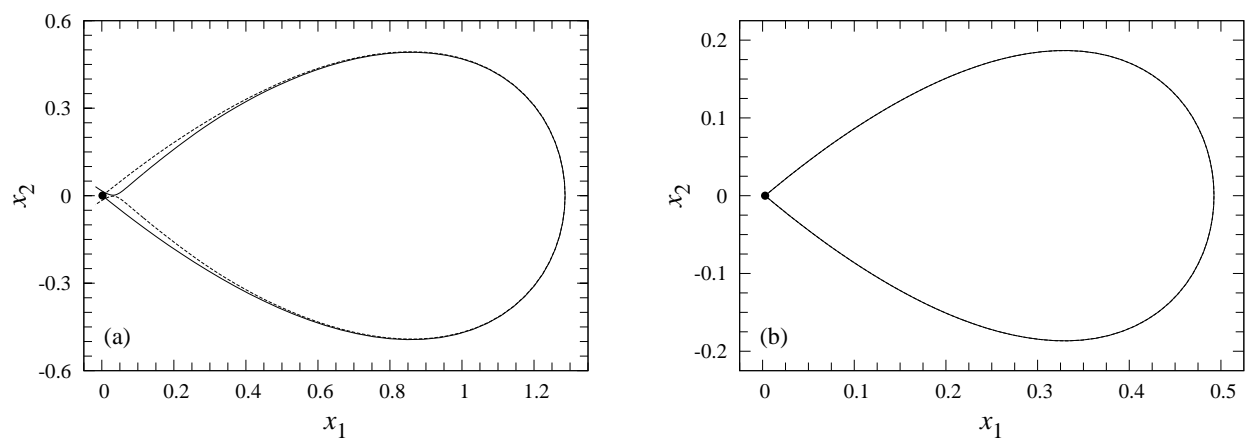

Figure B4: Stable and unstable manifolds of the outermost periodic orbits of Fig. B3 on the Poincaré section $\left\{y_{1}=0\right\}$ in (B.2): (a) Case (i); (b) case (ii). The solid and broken lines represent the stable and unstable manifolds, respectively. The point "•" represents the periodic orbits on the Poincaré section.

\section{Acknowledgments}

This work is partially supported by Ministry of Education, Science, Sports and Culture, Grant-in-Aid for Young Scientists (A) \#17684002, Grant-in-Aid for Exploratory \#19654014, and Grant-in-Aid for formation of COE at Hokkaido University; and by the Japan Society for the Promotion of Science, Grant-in-Aid for Scientific Research (C) \#18560056. The authors also thank Bart Oldeman for his advice in the modification of "AUTO97" to automatically compute the monodromy matrices of periodic orbits.

\section{References}

[1] V.I. Arnold, Mathematical Methods of Classical Mechanics, 2nd. ed. Springer-Verlag, New York, 1989. 
[2] V.I. Arnold, Ordinary Differential Equations, Springer-Verlag, New York, 1992.

[3] S. Boatto and H.E. Cabral, Nonlinear stability of a latitudinal ring of pointvortices on a nonrotating sphere, SIAM J. Appl. Math., 64 (2003), pp. 216230 .

[4] S. Boatto and J. Lasker, Point-vortex cluster formation in the plane and on the sphere: An energy bifurcation condition, Chaos, 13 (2003) pp. 824-835.

[5] H.E. Cabral, K.R. Meyer and D.S. Schmidt, Stability and bifurcation of the $N+1$ vortex problem on sphere, Regular Chaotic Dyn., 8 (2003) pp. 259282.

[6] A.R. Champneys and G.J. Lord, Computation of homoclinic solutions to periodic orbits in a reduced water-wave problem, Phys. D, 102 (1997), 232269.

[7] E. Doedel, A.R. Champneys, T.F. Fairgrieve, Y.A. Kuznetsov, B. Sandstede and X. Wang, AUTO97: Continuation and Bifurcation Software for Ordinary Differential Equations (with HomCont), 1997, available by anonymous ftp from ftp.cs. concordia.ca, directory pub/doedel/auto.

[8] J.R. Dormand and P.J. Prince, Practical Runge-Kutta processes, SIAM J. Sci. Stat. Comput. 10 (1989), pp. 977-989.

[9] M. T. DiBattista and L. M. Polvani, Barotropic vortex pairs on a rotating sphere, J. Fluid Mech., 358 (1998), pp. 107-133.

[10] D.G. Dritschel, The stability and energetics of co-rotating uniform vortices, J. Fluid Mech., 358 (1985), pp. 95-134.

[11] A. Goriely, Integrability and Nonintegrability of Dynamical Systems, World Scientific, New Jersey, 2001.

[12] C. Grotta-Ragazzo, Nonintegrability of some Hamiltonian systems, scattering and analytic continuation, Commun. Math. Phys., 166 (1994), pp. 255277.

[13] J. Guckenheimer and P.J. Holmes, Nonlinear Oscillations, Dynamical Systems, and Bifurcations of Vector Fields, Springer-Verlag, New York, 1983.

[14] E. Hairer, S.P. Nørsett, G. Wanner, Solving Ordinary Differential Equations I, 2nd ed., Springer-Verlag, Berlin, 1993.

[15] G. Haller, Chaos Near Resonance, Springer-Verlag, New York, 1999.

[16] M. Hénon and C. Heiles, The applicability of the third integral of motion: Some numerical experiments, Astron. J., 69 (1964), pp. 73-79.

[17] P.J. Holmes and J.E. Marsden, Melnikov's method and Arnol'd diffusion for perturbations of integrable Hamiltonian systems, J. Math. Phys., 23 (1982), pp. 669-675. 
[18] R. Kidambi and P.K. Newton, Motion of three point vortices on a sphere, Phys. D, 116 (1998), pp. 95-134.

[19] R. Kidambi and P.K. Newton, Collapse of three vortices on a sphere, Nouvo Cimento, 22C (1999), pp. 779-791.

[20] L.G. Kurakin, On nonlinear stability of the regular vortex systems on a sphere, Chaos, 14 (2004), pp. 592-602.

[21] L.M. Lerman, Hamiltonian systems with loops of a separatrix of a saddlecenter, Selecta Math. Sov., 10 (1991), pp. 297-306.

[22] C.C. Lim, Singular manifolds and quasi-periodic solutions of Hamiltonians for vortex lattices, Phys. D, 30 (1988), pp. 343-362.

[23] C.C. Lim, On singular Hamiltonians: the existence of quasi-periodic solutions and nonlinear instability, Bull. Amer. Math. Soc., 20(1) (1989), pp. 35-40.

[24] C.C. Lim, Existence of KAM tori in the phase-space of lattice vortex systems, J. Appl. Math. Phys. (ZAMP), 41 (1990), pp. 227-244.

[25] C.C. Lim, A combinatorial perturbation method and Arnold whiskered tori in vortex dynamics, Phys. D, 64 (1993), pp. 163-184.

[26] C.C. Lim, J. Montaldi, and M. Roberts, Relative equilibria of point vortices on the sphere, Phys. D, 148 (2001), pp. 97-135.

[27] C.C. Lim and J. Nebus, Vorticity, Statistical Mechanics, and Monte Carlo Simulation, Springer Monographs in Mathematics, Springer-Verlag, New York, 2006.

[28] K.R. Meyer and G.R. Hall, Introduction to Hamiltonian Dynamical Systems and the $N$-Body Problem, Springer-Verlag, New York, 1992.

[29] A. Mielke, P.J. Holmes and O. O'Reilly, Cascades of homoclinic orbits to, and chaos near, a Hamiltonian saddle-center, J. Dyn. Diff. Eqns., 4 (1992), pp. $95-126$.

[30] P.K. Newton, The N-vortex problem, Analytical techniques, SpringerVerlag, New York, 2001.

[31] P.K. Newton, The N-vortex problem on a rotating sphere: III. Ring configurations coupled to a background field, Proc. Roy. Soc. London Ser. A, 463 (2007), pp. 961-977.

[32] H.E. Nusse and J.A. Yorke, Dynamics: Numerical Explorations, 2nd ed., Springer-Verlag, New York, 1998.

[33] S. Pekarsky and J.E. Marsden, Point vortices on a sphere: Stability of relative equilibria, J. Math. Phys., 39 (1998), pp. 5894-5907. 
[34] L.M. Polvani and D.G. Dritschel, Wave and vortex dynamics on the surface of a sphere, J. Fluid Mech., 255 (1993), pp. 35-64.

[35] T. Sakajo, The motion of three point vortices on a sphere, Japan J. Indust. Appl. Math., 16 (1999), pp. 321-347.

[36] T. Sakajo, Motion of a vortex sheet on a sphere with pole vortices, Phys. Fluids, 16 (2004), pp. 717-727.

[37] T. Sakajo, Transition of global dynamics of a polygonal vortex ring on a sphere with pole vortices, Phys. D, 196 (2004), pp. 243-264.

[38] T. Sakajo, High-dimensional heteroclinic and homoclinic connections in odd point-vortex ring on a sphere, Nonlinearity, 19 (2005), pp. 75-93.

[39] T. Sakajo, Invariant dynamical systems embedded in the $N$-vortex problem on a sphere with pole vortices, Phys. D, 217 (2006), pp. 142-152.

[40] T. Sakajo, Erratum: Invariant dynamical systems embedded in the $N$-vortex problem on a sphere with pole vortices, Phys. D, 225 (2007), pp. 235-236.

[41] T. Sakajo, Integrable four-vortex motion on sphere with zero moment of vorticity, Phys. Fluids, 19(1) (2007), 017019.

[42] T. Sakajo and K. Yagasaki, Chaotic motion of the $N$-vortex problem on a sphere: II. Saddle centers in three-degree-of-freedom Hamiltonians, to appear in Phys. D (2007).

[43] S. Wiggins, Global Bifurcations and Chaos, Springer-Verlag, New York, 1988.

[44] S. Wiggins, Global Dynamics, Phase Space Transport, Orbits Homoclinic to Resonances, and Applications, American Mathematical Society, Providence, RI, 1993.

[45] S. Wiggins, Introduction to Applied Nonlinear Dynamical Systems and Chaos, 2nd ed., Springer-Verlag, 2003.

[46] K. Yagasaki, Horseshoes in two-degree-of-freedom Hamiltonian systems with saddle-centers, Arch. Rat. Mech. Anal., 154 (2000), pp. 275-296.

[47] K. Yagasaki, Homoclinic and heteroclinic orbits to invariant tori in multidegree-of-freedom Hamiltonian systems with saddle-centers, Nonlinearity, 18 (2005), pp. 1331-1350.

[48] K. Yagasaki, Higher-order Melnikov method and chaos for two-degree-offreedom Hamiltonian systems with saddle-centers, submitted for publication.

[49] K. Yagasaki, Numerical analysis for global bifurcations of periodic orbits in autonomous differential equations, in preparation. 
[50] K. Yagasaki, Numerical computation of homoclinic and heteroclinic orbits to nonhyperbolic trajectories, in preparation.

[51] K. Yagasaki, Numerical computation of stable and unstable manifolds of normally hyperbolic invariant manifolds, in preparation. 